\title{
VERGLEICH DER EIGENKAPITALAUSSTATTUNG DEUTSCHER, US-AMERIKANISCHER UND BRITISCHER UNTERNEHMEN*
}

\author{
Manfred Perlitz, Herbert Küpper, Helge Löbler**
}

\section{Inhaltsübersicht}

I. Einleitung

II. Grundlage der Untersuchung

III. Darstellung der Rechnungslegungsunterschiede

1. Sachanlagen

a) Neubewertung der Sachanlagen in Großbritannien

b) Abschreibungen

2. Immaterielles Anlagevermögen

3. Finanzanlagevermögen

4. Vorräte

5. Sonstiges Umlaufvermögen

6. Passivseite

IV. Berechnung der Eigenkapitalquoten

1. Ausgewiesenes Eigenkapital

2. Einfluß des Immateriellen Anlagevermögens, des "share of retained earnings“ und der Neubewertungsrücklage

3. Berücksichtigung der "deferred taxes"
a) Bilanziell ausgewiesene deferred taxes (DTA)
b) Potentielle deferred taxes (DTP)
c) Beispiel

4. Einfluss der FIFO- bzw. LIFO-Bewertung der Vorräte auf die Eigenkapitalquote

a) LIFO-Methode in den USA

b) Die Umrechnung der FIFO- auf die Durchschnittskostenmethode

V. Berücksichtigung der Pensionsrückstellungen

VI. Ergebnis 
* Wir danken dem Arbeitskreis Wirtschaft und Recht im Stifterverband für die deutsche Wissenschaft für die Finanzierung der Datenerhebung im Ausland sowie der Deutschen Forschungsgemeinschaft (DFG) für die weitere Finanzierung dieses Forschungsprojektes.

** Wir danken den Herren cand. rer. pol. Engelbert Götz und Christoph Weiser für ihre Unterstützung beim Erfassen und der Verarbeitung der Daten auf der EDV.

\section{I. $\quad$ Einleitung}

Die mangelhafte Eigenkapitalausstattung der deutschen Unternehmen und die Notwendigkeit einer Verbesserung dieser Situation gehören zu den derzeit meistdiskutierten wirtschaftlichen Themen in der Bundesrepublik ${ }^{1,2}$. Zugleich wird der Mangel an Eigenkapital für die Finanzierung von Innovationen im Hinblick auf die internationale Wettbewerbsfähigkeit der deutschen Unternehmen diskutiert ${ }^{3}$.

Dabei wird zum einen der stetige, trendmäßige Niedergang der vertikalen Eigenkapitalquoten deutscher Unternehmen seit 1965 (Beginn der Bundesbank-Statistik) beklagt ${ }^{4}$.

Bild 1 zeigt, daß von 1965 bis 1980 diese Quote von 29,8\% auf 20,9\% abgesunken ist.

Zum anderen wird in einer Reihe von Untersuchungen auf die im internationalen Vergleich ungünstige Eigenkapitalstruktur (vgl. Bild 2) deutscher Unternehmen hingewiesen ${ }^{5}$.

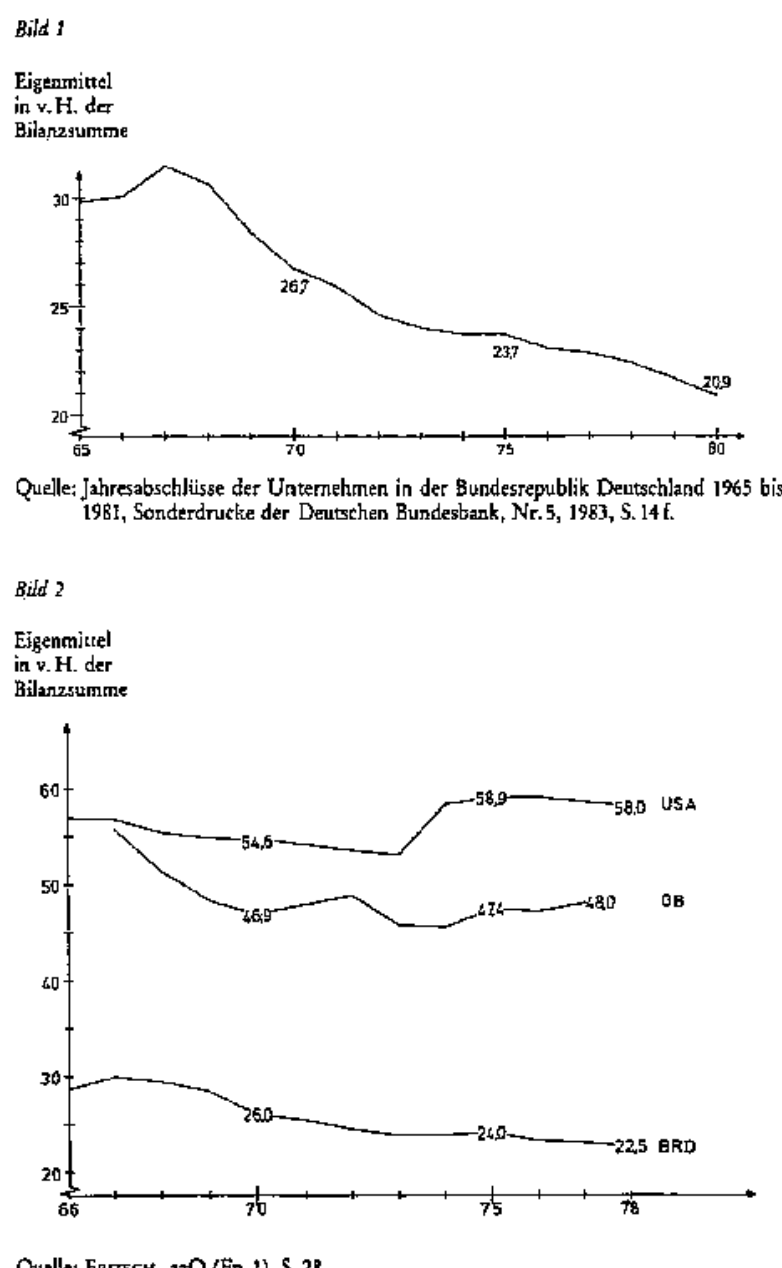

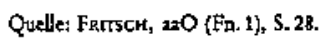


Bei Internationalen Vergleichen der Eigenkapitalausstattung von Unternehmen muß jedoch berücksichtigt werden, daß die Rechnungslegungsvorschriften aufgrund der vielfältigen Einflüsse des jeweiligen nationalen Gesellschafts- und Steuerrechts, der Struktur und Entwicklung der Kapitalmärkte, der Bilanzierungsgewohnheiten und der Standards der Wirtschaftsprüferorganisationen ${ }^{6}$ die Höhe des ausgewiesenen Eigenkapitals beeinflussen. Schlagworte wie "window dressing" in den USA und die Neigung zur Bildung „Stiller Reserven" in der Bundesrepublik verdeutlichen, daß ein unmittelbarer Vergleich der Kennzahl Eigenkapital/Gesamtkapital nur bedingt möglich ist.

Darüber hinaus wird die Interpretation der ausgewiesenen Eigenkapitalquote nicht nur von den unterschiedlichen Rechnungslegungsvorschriften, sondern auch von den verschiedenen sozio-ökonomischen Rahmenbedingungen beeinflußt. Dadurch kann die gleiche ökonomische Aktivität allein durch die unterschiedlichen Rechnungslegungsvorschriften bzw. gewohnheiten sowie durch die verschiedenen sozio-ökonomischen Rahmenbedingungen zu unterschiedlichen Darstellungen des wirtschaftlichen Geschehens führen ${ }^{7}$. Es ist daher scharf $\mathrm{zu}$ trennen zwischen dem Produktions-, Absatz-, Beschaffungs-, Investitions- und Finanzierungsprozeß mit den ihn beeinflussenden Faktoren und dem Abbildungsprozeß (vgl. Bild 3).

Bild 3

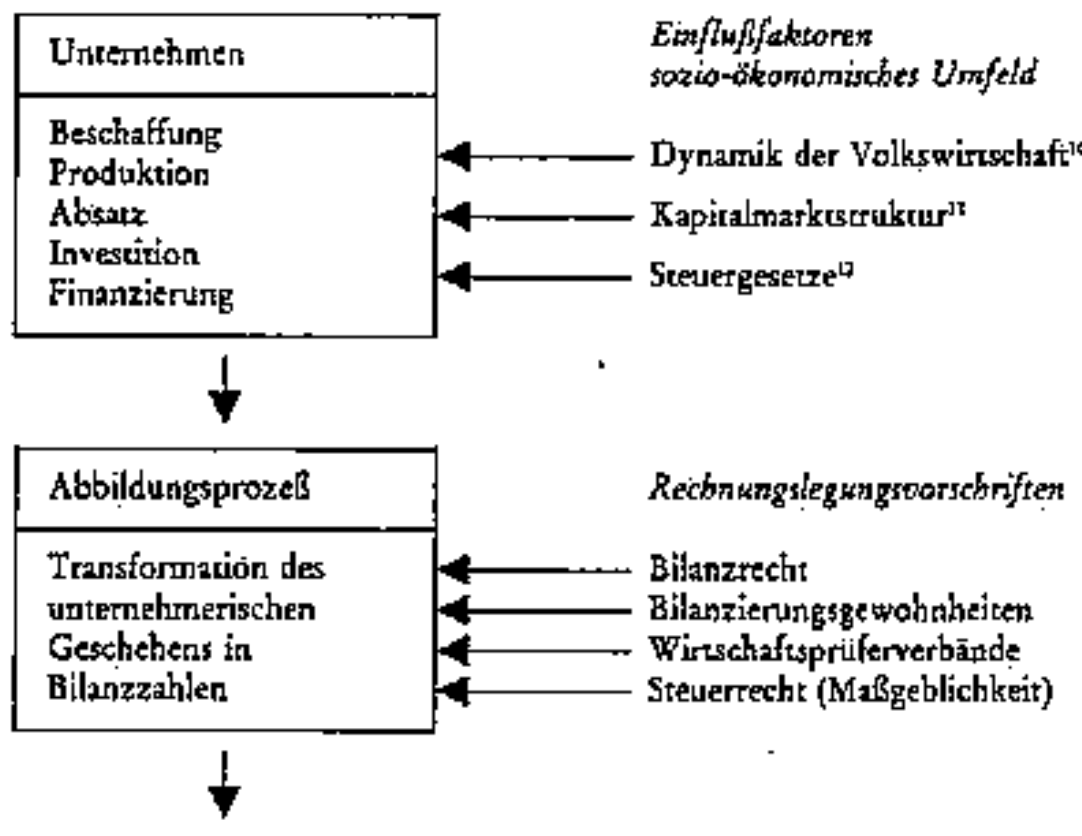

\begin{tabular}{|c|c|}
\hline \multicolumn{2}{|c|}{ Bilanx } \\
\hline SAV & \multirow{2}{*}{ EK } \\
\hline IAV & \\
\cline { 1 - 1 } FAV & \\
\cline { 1 - 1 } VORRATE & \multirow{2}{*}{ FK } \\
\cline { 1 - 1 } UV & \\
\hline
\end{tabular}

\author{
Abhk̈rzingèn: \\ SAV: Sachanlagevermögen \\ 1AV: Immacerielles Anlagevermöğen \\ FAV: Finanzanlagevermögen \\ UV: Untaufvermögen \\ EK: Eigertkapikal \\ FK: Frendkapiral
}


In den bisher vorliegenden Untersuchungen wurde regelmäßig nur der Zusammenhang zwischen Eigenkapitalquote und sozio-ökonomischem Umfeld analysierte, mit der expliziten oder impliziten Begründung, daß „die länderweisen Unterschiede z. T. so spektakulär (sind), daß abgrenzungs- und bewertungsrelevante Differenzen ihre Bedeutung mehr oder weniger einbüßen"“9.

Diese These, die für die Vergleichbarkeit der Datenbasis von eminenter Bedeutung ist, soll im folgenden überprüft werden. Die nachstehende Analyse untersucht die Auswirkungen der Unterschiede in den Ansatz- und Bewertungswahlrechten im Zusammenhang mit den Rechnungslegungsvorschriften der einzelnen betrachteten Länder auf die Eigenkapitalquote.

Dabei werden zuerst die nationalen Unterschiede in der Rechnungslegung für die wichtigsten Bilanzpositionen einzeln untersucht (Kapitel III). Anschließend wird die Auswirkung dieser Unterschiede auf den Eigenkapitalausweis und damit auf die Eigenkapitalquote anhand von Modellrechnungen und Simulationen analysiert (Kapitel IV). In Kapitel V wird der Zusammenhang zwischen der länderweise unterschiedlichen Behandlung des Pensionsaufwandes und der Eigenkapitalquote dargestellt. Die wichtigsten Ergebnisse werden dann in Kapitel VI erörtert.

\section{Grundlage der Untersuchung}

Basis der vorliegenden Untersuchung sind die Jahresabschlüsse von 189 USamerikanischen, 196 britischen und 140 deutschen Unternehmen (im folgenden Sample genannt) der größten 100 und aus der Gruppe der zwischen Rang 400 und 500 liegenden größten Unternehmen der Jahre 1979 und 1980. Außerdem wurden die Daten der Bonner Stichprobe für 295 deutsche Unternehmen verwendet; jedoch wurden hier aus Vergleichsgründen nur die Einzelabschlüsse deutscher Industrieaktiengesellschaften berücksichtigt.

Die Verteilung der Unternehmen nach Größenklassen ist Tabelle 1 zu entnehmen.

\begin{tabular}{llcc} 
Tabelle 1 & & \multicolumn{2}{c}{ Gröfeniklasse } \\
\hline Land & Jahr & $1-100$ & $400-500$ \\
\hline Deukschland & & 95 & 35 \\
Sampie & 1979 & 100 & 40 \\
\hline Grobbritunnien & 1980 & 98 & 91 \\
& 1979 & 98 & 98 \\
\hline USA & 1980 & 98 & 94 \\
& 1979 & 95 & 96 \\
\hline
\end{tabular}

Um die Repräsentanz der deutschen Stichprobe zu überprüfen, sind die wichtigsten Bilanzpositionen der Abschlüsse von 1980 den Bilanzstrukturzahlen der Kapitalgesellschaften aus der Bilanzstatistik der Deutschen Bundesbank gegenübergestellt worden (vgl. Tabelle 2) ${ }^{13}$.

\begin{tabular}{|c|c|c|}
\hline Bil anzposition & $\begin{array}{l}\text { Anteile } \\
\text { Sample }\end{array}$ & $\begin{array}{l}\text { Bilarzsummen } \\
\text { Bundesbankstatistik }\end{array}$ \\
\hline $\begin{array}{l}\text { SAV (incl. IAV) } \\
\text { FAV } \\
\text { YORRÄTE } \\
\text { UV (incl. Kasse, Wertpapiere u. RAP) } \\
\text { EK } \\
\text { RAtckstellungen } \\
\text { sonst. Verb. }\end{array}$ & $\begin{array}{r}29,7 \\
7,7 \\
26,8 \\
35,8 \\
26,1 \\
21,4 \\
52,5\end{array}$ & $\begin{array}{r}28,8 \\
8,6 \\
22,0 \\
40,3 \\
25,1 \\
21,0 \\
53,4\end{array}$ \\
\hline
\end{tabular}


Außer den Verschiebungen zwischen Vorrats- und Umlaufvermögen bestehen zwischen den beiden Stichproben kaum nennenswerte Unterschiede. Das verwendete Sample kann also durchaus als repräsentativ für die deutschen Kapitalgesellschaften angesehen werden.

Die Eigenkapitalquote der US-amerikanischen Unternehmen beträgt im Sample 46,6 \%, in der Statistik bei Gruhler 49,8 $\%^{14}$. Es handelt sich bei Gruhler um industrielle Aktiengesellschaften, deren Eigenkapitalquote er für deutsche Unternehmen mit 30,4\% angibt, also auch um einige Prozentpunkte höher als in unserem Sample.

Für Großbritannien gibt Gruhler die Eigenkapitalquote (nur größere Gesellschaften) mit 43,5\% an, in unserem Sample beträgt sie 44,2 \%.

Die Eigenkapitalquoten der im Sample untersuchten Unternehmen stimmen also mit denen allgemein verbreiteter Statistiken überein und dürften damit für das Untersuchungsziel eine ausreichende Repräsentanz besitzen.

\section{Darstellung der Rechnungslegungsunterschiede}

Die wichtigsten Aktivpositionen der deutschen Bilanz sind nach dem Gliederungsschema des $\S 151$ AktG:

- Sachanlagen

- Immaterielles Anlagevermögen

- Finanzanlagen

- Vorräte

- Andere Gegenstände des Umlaufvermögens.

Im folgenden werden die Bilanzierungs- und Bewertungsunterschiede der einzelnen Länder für jede der genannten Positionen untersucht.

\section{Sachanlagen}

Das Sachanlagevermögen stellt mit 29,4 \% der Bilanzsumme die zweitgrößte Einzelposition der deutschen Bilanz dar (USA 39,4 \%; GB 33,8 \%). Die Grundsätze der Bilanzierung sind in $\S 153$ Abs. 1 Satz 1 AktG geregelt: „Gegenstände des Anlagevermögens sind zu den Anschaffungs- oder Herstellungskosten vermindert um Abschreibungen oder Wertberichtigungen nach $\S 154$ anzusetzen".

Zwar gilt dieser Grundsatz der Bewertung zu Anschaffungskosten als "historical cost accounting" im Prinzip auch in den USA und Großbritannien, aber eine wichtige britische Besonderheit ist die sog. Neubewertung (revaluation) der nicht abnutzbaren und der besonders langlebigen Teile des Anlagevermögens, die im folgenden dargestellt wird ${ }^{15}$.

\section{a) Neubewertung der Sachanlagen in Großbritannien}

Das Problem einer Inflationsbereinigung in der Bilanz wurde zwar in Deutschland ausgiebig diskutiert $^{16}$, hat aber in den Rechnungslegungsvorschriften bisher nur peripher einen Niederschlag gefunden ${ }^{17}$. In den USA sind Ergänzungsrechnungen vorgeschrieben ${ }^{18}$, und auch in Großbritannien müssen die Aktiengesellschaften seit 1.1.1980 eine Nebenrechnung mit "current cost accounting" veröffentlichen ${ }^{19}$.

Darüber hinaus ist es seit 1960 für britische Unternehmen üblich geworden, in bestimmten Abständen die nicht abnutzbaren und besonders langlebigen Teile des Sachanlagevermögens zu Wiederbeschaffungspreisen neu zu bewerten. 
Der Betrag, um den das Sachanlagevermögen höher bewertet wird (revaluation surplus), ist einer nichtausschüttbaren Rücklage und damit dem Eigenkapital zuzuführen.

Von den 196 untersuchten britischen Unternehmen haben $127(=65 \%)$ in den letzten zwanzig Jahren zumindest eine Neubewertung vorgenommen. Da die britischen Unternehmen nicht die gesamte Position „Rücklagen", sondern lediglich die Zuführung erläutern müssen, ließen sich in der vorliegenden Untersuchung nur bei 51 Unternehmen der Gesamtbetrag der Höherbewertung und bei weiteren 37 Unternehmen der Zuwachs des Jahres 1980 ermitteln. Für die übrigen 39 Unternehmen konnten keine genauen Daten über die Neubewertungsrücklage gewonnen werden, da sie vor 1979 gebildet wurde.

Die offengelegten „Neubewertungsrücklagen" machen insgesamt 2,63\% der Bilanzsumme des Rechnungsjahres 1980 aus. Schätzungen aus weiteren Bilanzerläuterungen lassen schließen, daß mit hoher Wahrscheinlichkeit die Neubewertungsrücklage mehr als 3,6\% der Bilanzsumme beträgt ${ }^{20}$.

\section{b) Abschreibungen}

In Deutschland gilt das sogenannte Maßgeblichkeitsprinzip der Handelsbilanz für die Steuerbilanz. Umgekehrt werden durch steuerrechtliche Vorschriften bestimmte Abschreibungen steuerlich nur dann anerkannt, wenn sie auch in der Handelsbilanz vorgenommen werden (umgekehrtes Maßgeblichkeitsprinzip) ${ }^{21}$. Ein solches Prinzip für die Rechnungslegungsvorschriften gibt es in den USA und Großbritannien nicht. Dies hat dazu geführt, daß $95 \%$ der untersuchten US-amerikanischen und etwa $98 \%$ der britischen Unternehmen in der Handelsbilanz linear abgeschrieben, steuerlich jedoch höhere Abschreibungsbeträge geltend gemacht haben, in Deutschland hingegen haben fast alle Unternehmen degressiv abgeschrieben.

Die Behandlung der außerplanmäßigen Abschreibungen ist für Deutschland in $\S 154$ Abs. 2 AktG geregelt.

Satz 1 Nr. 1 gestattet die Vornahme von Abschreibungen wegen außerplanmäßiger, technisch oder wirtschaftlich bedingter Wertminderung (bei voraussichtlich dauernder Wertminderung müssen sie durchgeführt werden); Satz $1 \mathrm{Nr} .2$ erlaubt die Abschreibung auf den steuerlich zulässigen niedrigeren Wert. Die meisten außerplanmäßigen Abschreibungen deutscher Unternehmen basieren auf steuerlichen Sonderabschreibungen (z.B. nach Berlinförderungsgesetz, $§ 6 \mathrm{~b}$ EStG oder $§ 7 \mathrm{~d}$ EStG [Umweltschutzmaßnahmen]) ${ }^{22}$.

Wegen der Unabhängigkeit von Handels- und Steuerbilanz sind in den USA und Großbritannien außerordentliche Abschreibungen in der Handelsbilanz nur wegen technischer oder wirtschaftlicher Wertminderung zulässig. Somit können in der Handelsbilanz keine aus steuerlichen Gründen vorgenommenen Sonderabschreibungen geltend gemacht werden.

Die Festlegung der Nutzungsdauer von Sachanlagevermögen ist in den einzelnen betrachteten Ländern nicht generell geregelt. Die Unterschiede lassen sich für den externen Bilanzanalytiker auch aus den veröffentlichten Geschäftsberichten nicht im Detail ermitteln.

Anhaltspunkte für die Unterschiede in den Nutzungsdauern könnte der Quotient SAV/AfA liefern.

Für die untersuchten Jahresabschlüsse ergeben sich die in Tabelle 3 dargestellten geschätzten Nutzungsdauern.

Tabeile J: Geschăczte Nutzungsdauetn (iin Jahren)

\begin{tabular}{|c|c|c|c|c|c|}
\hline Jahkr & Land & $\begin{array}{l}\text { Deutschland } \\
N=140\end{array}$ & $\begin{array}{c}\text { Deutschland } \\
\mathrm{N}=295\end{array}$ & $\begin{array}{c}\text { Großsbritannien } \\
\mathrm{N}=196\end{array}$ & $\begin{array}{c}\text { USA } \\
N=189\end{array}$ \\
\hline $\begin{array}{l}1979 \\
1980\end{array}$ & & $\begin{array}{l}5,7 \\
5,6\end{array}$ & $\begin{array}{l}6,4 \\
6,1\end{array}$ & $\begin{array}{l}13,1 \\
12,9\end{array}$ & $\begin{array}{l}9,6 \\
9,5\end{array}$ \\
\hline
\end{tabular}


Die dort wiedergegebenen Jahreszahlen ergeben nur Anhaltspunkte für die Unterschiede in den Nutzungsdauern, da sich in ihnen auch die verschiedenen Abschreibungsmethoden und Investitionsmöglichkeiten niederschlagen.

Wenn auch die Wirkungen der einzelnen Parameter auf die Abschreibungen (Abschreibungsmethode, Sonderabschreibungen, Nutzungsdauern) nicht isoliert ermittelt werden können, so läßt sich doch der Gesamteffekt aus den Abschreibungsunterschieden zwischen den einzelnen Ländern berechnen.

Ausgangspunkt für diese Analyse ist die Position "deferred taxes". Sie ist im wesentlichen das Korrektiv für die unterschiedliche Behandlung der Abschreibungen in Handels- und Steuerbilanz in den USA und Großbritannien ${ }^{23}$.

Das Prinzip der "deferred taxes" soll im folgenden bezogen auf die Abschreibungen dargestellt werden:

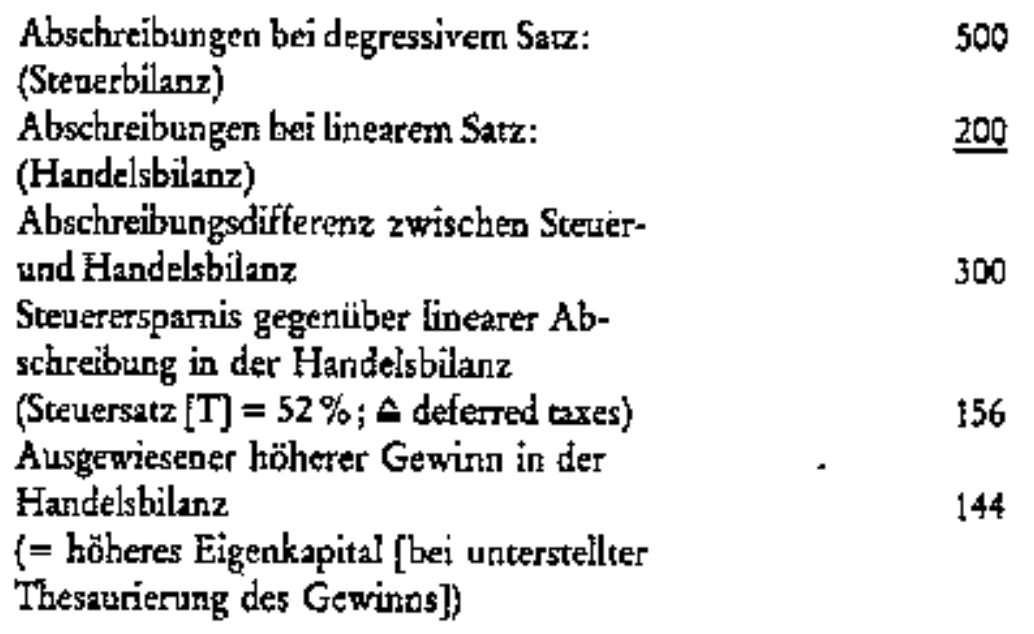

Während in britischen Geschäftsberichten eine Trennung der Position "deferred taxes" in den Abschreibungs-, other timing differentes- und stock appreciation relief-Effekt erfolgt, ist eine solche Aufschlüsselung in den USA nicht üblich.

Zur Erläuterung der Auswirkungen der "deferred taxes" auf die Gewinn- und Eigenkapitalentwicklung dient das folgende Beispiel:

Ein Unternehmen erwerbe eine Maschine für 100000 DM, die eine erwartete Nutzungsdauer von 10 Jahren und einen Restwert von $0 \mathrm{DM}$ besitze. Das Unternehmen habe einen jährlichen Gewinn vor Abschreibung und Steuern von 100000 DM.

Tabelle 4 zeigt die Abschreibungen (Zeile 2) und den Gewinn nach Steuern (Zeile 5) unter der Annahme, daß eine geometrisch-degressive Abschreibung nach $\S 7$ Abs. 2 Satz 2 EStG vorgenommen wird. Der anzuwendende Prozentsatz darf höchstens das dreifache des bei linearer Abschreibung in Betracht kommenden Prozentsatzes betragen und $30 \%$ nicht übersteigen ${ }^{24}$.

Tabelle 4: Abxchreibungen in der Bundesrepublik Deutschland

\begin{tabular}{|c|c|c|c|c|c|c|c|c|c|c|c|c|}
\hline \multirow[b]{2}{*}{ Zeile } & \multirow[b]{2}{*}{ Position } & \multicolumn{7}{|c|}{ Jahr } & \multirow[b]{2}{*}{$B$} & \multirow[b]{2}{*}{9} & \multirow[b]{2}{*}{10} & \multirow[b]{2}{*}{ Zeile } \\
\hline & & $\mathbf{I}$ & 2 & 3 & 4 & 5 & 6 & 7 & & & & \\
\hline 1 & $\begin{array}{l}\text { Struespflichtiges Einkom- } \\
\text { metn vor Abschrribungen } \\
\text { und Ertrapstevtre }\end{array}$ & $100000,-$ & 100000 & $100000,-$ & $100000,-$ & $100000,-$ & $100000,-$ & $100000_{--}$ & 100000 & 100000 & 100000, & $!$ \\
\hline $\begin{array}{l}2 \\
3\end{array}$ & $\begin{array}{l}\text { Degressive Abschreibung } \\
\text { Steuerplitichtiges Ein- } \\
\text { lommer }\end{array}$ & $\begin{array}{l}30000_{-}^{-} \\
70000_{-}^{-}\end{array}$ & $\begin{array}{l}21000_{1}- \\
79000_{2}-\end{array}$ & $\begin{array}{l}147000_{1}- \\
85300^{2}\end{array}$ & $\begin{array}{l}10290,- \\
89710,-\end{array}$ & $\begin{array}{l}7275,- \\
92797,-\end{array}$ & $\begin{array}{r}5042,10 \\
94957,90\end{array}$ & $\begin{array}{r}3529,47 \\
96+70,53\end{array}$ & $\begin{array}{r}2745,54 \\
97254,86\end{array}$ & $\begin{array}{r}2745,14 \\
97254,86\end{array}$ & $\begin{array}{r}2745,15 \\
97254,85\end{array}$ & 2 \\
\hline $\begin{array}{l}1 \\
5\end{array}$ & $\begin{array}{l}\text { Körperschaftstesuer }(\$ 2 \%) \\
\text { Getwinn nach Stetuern }\end{array}$ & $\begin{array}{l}36400,- \\
33600_{1}-\end{array}$ & $\begin{array}{l}41080,- \\
37920 .-\end{array}$ & $\begin{array}{l}41356_{1}- \\
40944_{1}-\end{array}$ & $\begin{array}{l}46649,20 \\
13060,80\end{array}$ & $\begin{array}{l}48254,44 \\
44512,56\end{array}$ & $\begin{array}{l}49378,10 \\
45579,80\end{array}$ & $\begin{array}{l}50164,67 \\
46305,96\end{array}$ & $\begin{array}{l}\times 0572,53^{-} \\
16682,33\end{array}$ & $\begin{array}{l}505 \pi 2,53 \\
16682,33\end{array}$ & $\begin{array}{l}50572,53 \\
46682,31\end{array}$ & 4 \\
\hline 6 & Xumslierec Gesinn & $33600,-$ & $71520,-$ & $112464,-$ & 155524,80 & 200067,36 & 245647,36 & 291953,02 & 338635,35 & 385313,68 & $432000,-$ & 6 \\
\hline 7 & $\begin{array}{l}\text { SAV abzgl, kumulier1er Ab- } \\
\text { schreibungen (sog. Restwert) }\end{array}$ & 70000 & 49000 & $34300_{1}-$ & $21010,-$ & $16807,-$ & 11764,90 & 8235,13 & 5490,29 & 2745,15 & - & 7 \\
\hline
\end{tabular}


Tabelle 5 stellt die Bilanzierung nach US-amerikanischem oder britischem Recht dar, wenn in der Handelsbilanz linear (Zeilen 2 und 3) und in der Steuerbilanz degressiv (Zeile 4) 25 abgeschrieben wird. Bei einem Steuersatz von $52 \%$ und einem Gewinn vor Steuern von 90000 DM beträgt der GuV-Steueraufwand jährlich 46800 DM (Tabelle 5, Summe der Zeilen 4-6). In den vier ersten Jahren müssen aufgrund der steuerlich relevanten degressiven Abschreibungen weniger als 46800 DM bezahlt werden (Zeile 4), der Differenzbetrag wird passivisch abgegrenzt und in die "deferred taxes" eingestellt (Zeile 5). Im fünften Jahr kehrt sich der Prozeß um, das steuerpflichtige Einkommen (der Steuerbilanz; Tabelle 4, Zeile 3) ist größer als der Gewinn vor Steuern in der Handelsbilanz (Tabelle 5, Zeile 3), und die "deferred taxes" werden wieder aufgelöst.

\begin{tabular}{|c|c|c|c|c|c|c|c|c|c|c|c|c|}
\hline \multirow[b]{2}{*}{ Zilc } & \multirow[b]{2}{*}{ Pcairjar } & \multirow[b]{2}{*}{1} & \multirow[b]{2}{*}{2} & \multirow[b]{2}{*}{3} & \multicolumn{2}{|r|}{ Jath } & \multirow[b]{2}{*}{6} & \multirow[b]{2}{*}{7} & \multirow[b]{2}{*}{8} & \multirow[b]{2}{*}{9} & \multirow[b]{2}{*}{10} & \multirow[b]{2}{*}{ Zeike } \\
\hline & & & & & $i$ & 5 & & & & & & \\
\hline 1 & $\begin{array}{l}\text { Gewian wor Abschtreibungen } \\
\text { und Ertratulouert }\end{array}$ & 10000 - & $100000_{--}$ & $100000,-$ & $100000,-$ & 10000 & $1000000_{-}-$ & $1000000,-$ & $1000001,-$ & $100000_{-}-$ & $100 \infty \infty_{r}$ & 1 \\
\hline 2 & Lineare Abechreibing & 10000 & $100000_{1-}$ & too00,- & $1000,-$ & $10000,-$ & $10000-$ & $10000_{-}^{-}$ & $20000,-$ & $10 \log _{1-}-$ & $10000_{1-}$ & 2 \\
\hline 3 & Gevinn wor staxti & $900000-$ & $\infty 0+0,-$ & $90000-$ & प्रODOC, & $5000-$ & $90000,-$ & 90004 & $90000,-$ & $90000,-$ & $\infty \infty_{-1}$ & 3 \\
\hline 4 & 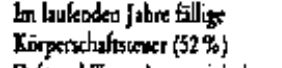 & $36+000_{1}^{-}$ & $41000-$ & $43 b_{\text {- }}$ & 46649,20 & 48254,41 & 4937, 10 & 50164,67 & 50577,53 & 5057,53 & 50572,53 & $\begin{array}{l}\text { Sieveranfivand } \\
\text { i. d, Getwith- }\end{array}$ \\
\hline 5 & 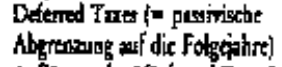 & $10400,-$ & 5720. & $244,-$ & 150,80 & - & - & - & - & - & - & $\begin{array}{l}\text { und Verhat: } \\
\text { rtchloung! }\end{array}$ \\
\hline 6 & Aufósung der "Defernal Taxes" & - & - & - & - & $(1454,4)$ & {$[258,10)$} & $(3364,67)$ & $\{m, 53\}$ & $(377,53)$ & $\{37 \cap 5,5\}$ & jarr. $4680,-$ \\
\hline$?$ & Geving naxh Sicterta & $43200-$ & $132000-$ & $43200,-$ & $41200,-$ & $132002-$ & $+3200 .-$ & $+3200-$ & $4200,-$ & $43 \mathrm{XO}_{-}-$ & $43200=$ & 7 \\
\hline 8 & Kropulinte Defkred 'Taxs & $10+04-$ & $161200_{2}-$ & $1 \pm 54,-$ & 18714,80 & 17260.36 & $196: 2,26$ & 11317,59 & 7545,06 & 572,53 & - & f \\
\hline 9 & Remulietur $G_{t \text { minn }}$ & (3) $200-$ & 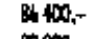 & $129600 .-$ & $172800,-$ & 2160000 & $259200 .-$ & $302400,-$ & $345600,-$ & $388800-$ & $\$ \$ 2000,-$ & 9 \\
\hline 10 & Sudanlagevemö̈gen (Restwer) & $90000-$ & $\cos _{1}-$ & $70000,-$ & $\mathrm{COCOMO}_{3}-$ & $50000,-$ & $40000,-$ & $30004-$ & $2000,-$ & $10000-$ & - & 10 \\
\hline 11 & $\begin{array}{l}5 A V_{L S A}-S A V_{\text {BRD }} \\
=\frac{1}{D S S} \times \text { Deferted TaRT }\end{array}$ & $20004-$ & $31000,-$ & $35700-$ & $35990-$ & $33193,-$ & 2825,10 & 2176,57 & 1450971 & $725+, 85$ & - & !] \\
\hline 12 & 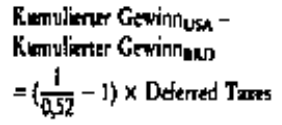 & $9600,-$ & $14800,-$ & $17136,-$ & 17275,20 & 55937,64 & 1355231 & 10446,98 & $6 \%+, 65$ & 3487,37 & - & 12 \\
\hline
\end{tabular}

Die Höhe der zu ermittelnden Eigenkapitalquote wird entscheidend durch die kumulierten Nettogewinne (unter Annahme der Thesaurierung) und den Restwert des Anlagegutes bestimmt. Tabelle 4, Zeilen 6 und 7 bzw . Tabelle 5, Zeilen 9 und 10 zeigen, daß der Gewinn und das Sachanlagevermögen in den USA und Großbritannien bis zur vollständigen Abschreibung im zehnten Jahr erheblich über den entsprechenden deutschen Werten liegen. Tabelle 5, Zeile 11 stellt die kumulierten Differenzen zwischen den deutschen und USamerikanischen bzw. britischen Restwerten des Anlagegutes dar. Zeile 12 zeigt die kumulierten Differenzen zwischen den Gewinnen.

Das Beispiel macht deutlich, daß aus den "deferred taxes" die entsprechenden Einflüsse auf den Gewinn und die Höhe des Sachanlagevermögens im Unterschied zu der deutschen Rechnungslegung anhand der Zeilen 11 und 12 bestimmt werden können. Die Möglichkeiten dieser Ermittlung werden im Kapitel IV näher dargestellt.

Die Bedeutung der Position "deferred taxes" geht daraus hervor, daß sie im Durchschnitt 4,4 \% der Bilanzsumme der US-amerikanischen Unternehmen ausmacht.

Eine Besonderheit der britischen Bilanzen ist die Unterscheidung zwischen "bilanziell ausgewiesenen" und ,potentiellen deferred taxes". In der Bilanz auszuweisen sind nur die "deferred taxes", die in den nächsten drei Jahren aufgelöst werden müssen (bilanziell ausgewiesene deferred taxes). Nicht auszuweisen sind diejenigen "deferred taxes", für die die Geschäftsleitung nachweisen kann, daß sie in den nächsten drei Jahren nicht aufgelöst werden müssen und für die auch darüber hinaus nicht mit einer Auflösung zu rechnen ist (potentielle deferred taxes $)^{26}$. Damit sind diese Bestandteil des Gewinns nach Steuern und somit 
Eigenkapital. Eine Auflösung der ,potentiellen deferred taxes" ist dann nicht vorzunehmen, wenn die Zuführung zu den "deferred taxes" größer oder gleich der Auflösung ist.

Die „,bilanziell ausgewiesenen deferred taxes" machen in Großbritannien $2 \%$, die „potentiellen deferred taxes" 4,7 \% der Bilanzsumme aus.

Eine weitere Besonderheit ist die großzügige steuerliche Abschreibung.

Tabelle 6 zeigt, daß z.B. für Industriegebäude eine steuerliche Abschreibung von $75 \%$, für Maschinen sogar von $100 \%$ zulässig ist.

\begin{tabular}{|c|c|c|c|}
\hline Xutegorien & $\begin{array}{l}\text { initizl } \\
\text { allowance }\end{array}$ & $\begin{array}{l}\text { first-year } \\
\text { allowance }\end{array}$ & $\begin{array}{l}\text { writing-down } \\
\text { allowance }\end{array}$ \\
\hline & bis z.u ... v. $\mathrm{H}_{1}$ & bis $2 \mathrm{u} \ldots \mathrm{v}, \mathrm{H}$ & mit ... v. H. \\
\hline $\begin{array}{l}\text { Gewerbliche Gebăude } \\
\text { Hotels } \\
\text { Merchinen, maschinelle Anlagen, sonstige } \\
\text { Besriebsvorrichrungen } \\
\text { Bergbau, Erdöl } \\
\text { Wirtschaftsgütter, die der wissenschaftlichen } \\
\text { Forschung dienen } \\
\text { Erworbene Patente } \\
\text { Erworbenes industrieltes Know-how }\end{array}$ & $\begin{array}{ll}75 & (2) \\
20 & (2)\end{array}$ & $\begin{array}{r}100 \\
40 \quad(b) \\
100\end{array}$ & 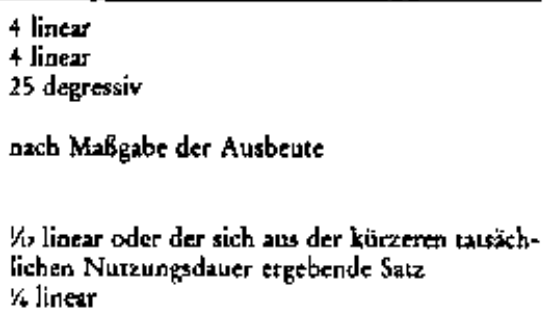 \\
\hline
\end{tabular}

2) $100 \%$ in "enterprise zones" mit $25 \%$ linearer Abschreibung

b) Freic Abschreibung in Entwicklungszonen und Nord-Ir]and

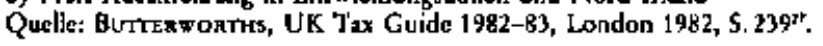

\section{Immaterielles Anlagevermögen}

Die Behandlung des Immateriellen Anlagevermögens ist in den betrachteten Ländern sehr unterschiedlich. Von den untersuchten Unternehmen aktivieren in den USA 45,3\% Immaterielles Anlagevermögen, in Deutschland $71 \%$. In Großbritannien berichten $70 \%$ der Unternehmen über das Immaterielle Anlagevermögen, viele setzen diese Position jedoch direkt von den Rücklagen ab und lediglich $60 \%$ führen eine Aktivierung durch.

Der Anteil des aktivierten Immateriellen Anlagevermögens an der Bilanzsumme beträgt in Deutschland durchschnittlich 0,33\%, in Großbritannien 2,57\% und in den USA 2,42\%.

Unterschiede ergeben sich beispielsweise für entgeltlich erworbene immaterielle Anlagewerte, für die in den USA grundsätzlich eine Aktivierungspflicht, in Deutschland und Großbritannien ein Ansatzwahlrecht besteht ${ }^{28}$.

Für selbsterstelltes Immaterielles Anlagevermögen existiert in den USA ein Aktivierungswahlrecht (außer für die Kosten der Forschung und Entwicklung), in den beiden anderen Ländern ist die Aktivierung untersagt.

Aufwendungen, die mit der Gründung des Unternehmens bzw. der Kapitalbeschaffung verbunden sind, dürfen in den USA und Großbritannien aktiviert werden, jedoch nicht in Deutschland (außer den Ingangsetzungskosten) ${ }^{29}$. Die Vergleichbarkeit der Position Immaterielles Anlagevermögen wird durch die unterschiedliche Handhabung der Abschreibungen in den einzelnen Ländern beeinträchtigt. So ist der „entgeltlich erworbene Geschäftswert (goodwill)" in Deutschland gem. § 153 Abs. 5 Satz 2 AktG innerhalb von fünf Jahren mit jährlich mindestens $20 \%$, in den USA über einen angemessenen Zeitraum (40 Jahre dürfen nicht überschritten werden) 30 abzuschreiben. In Großbritannien gibt es keine Vorschrift, welche den Abschreibungszeitraum festlegt.

Der Vergleich der unterschiedlichen Komponenten des Immateriellen Anlagevermögens ist nicht unmittelbar möglich, da nicht erkennbar ist, wieviel Immaterielles Anlagevermögen aktiviert und wieviel in die ,Stillen Reserven" überführt worden ist. 
Um die Unterschiede zwischen den einzelnen Ländern anzugleichen, kann man unterstellen, daß die Unternehmen immaterielle Anlagegüter nicht aktivieren und sich somit wie ein „,vorsichtiges" deutsches Unternehmen verhalten.

\section{Finanzanlagevermögen}

Zum Finanzanlagevermögen zählen gem. § 151 Abs. 1 AktG Beteiligungen, Wertpapiere des Anlagevermögens und Ausleihungen mit einer Mindestlaufzeit von vier Jahren. Ihm entspricht in den US-amerikanischen und britischen Bilanzen die Position "investments", die jedoch

zusätzliche Elemente enthält (z.B. Lebensversicherungen, Forderungen an Konzernunternehmen, etc.).

Das Finanzanlagevermögen in den deutschen Bilanzen beträgt 7,7\% der Bilanzsumme (USA $6,1 \%$; GB 2,6\%).

Der größte Unterschied besteht in der Bilanzierung der $20 \%$-50 \%igen Beteiligungen.

Diese werden in Deutschland in der Regel ${ }^{31}$ nicht konsolidiert, sondern zu Anschaffungskosten bewertet.

In den USA und im britischen Gruppenabschluß werden diese sogenannten "associated companies" hingegen nach der Equity-Methode bilanziert. Danach werden die Beteiligungen zuerst zu Anschaffungskosten bewertet und in jeder Periode um die anteiligen Gewinne bzw. Verluste (abzüglich der ausgeschütteten Dividende) korrigiert ${ }^{32}$.

In den britischen Gruppenabschlüssen wird das "Gesamtinteresse" an der "associated company" folgendermaßen bilanziert:

1. Anschaffungskosten (abzüglich Wertberichtigung) plus

2. Anteil an den (nach Erwerb) entstandenen Rücklagen des assoziierten Unternehmens (sog. share of retained earnings of associated companies) $)^{33}$.

Dieser "share of retained earnings of associated companies" betrug bei den britischen Unternehmen $43 \%$ des Finanzanlagevermögens. Dieser Betrag führt in Deutschland bei den $20 \%-50 \%$ igen Beteiligungen zu „Stillen Reserven". In den amerikanischen Geschäftsberichten wird über den "share of retained earnings of associated companies" nicht informiert.

\section{Vorräte}

Das Vorratsvermögen macht in Deutschland 26,8 \% der Bilanzsumme aus (USA $22 \%$; GB $30,5 \%$ ). Nach deutschem Aktienrecht muß eine Aktivierung der Vorräte auf der Basis von Anschaffungs- und Herstellungskosten erfolgen, es sei denn, es ergäbe sich am Bilanzstichtag ein niedrigerer beizulegender Wert (strenges Niederstwertprinzip). Grundsätzlich muß dabei für jeden Gegenstand eine Einzelbewertung vorgenommen werden, in Ausnahmefällen kann jedoch eine Gruppenbewertung (Sammelbewertung) erfolgen.

Bei der Ermittlung der Herstellungskosten besteht ein Bewertungswahlrecht, das Ansätze zwischen den variablen und vollen Herstellkosten zuzüglich der Verwaltungskosten erlaubt ${ }^{34}$. In Großbritannien gibt es eine ähnliche Regelung, wobei die allgemeinen Verwaltungskosten nicht aktivierungsfähig sind. Die USamerikanischen Regelungen stimmen im Kern mit den britischen überein ${ }^{35}$.

Das Niederstwertprinzip gilt für alle Länder, jedoch ergeben sich einige Besonderheiten hinsichtlich der Fortführung der niedrigeren Werte und des Wahlrechts gem. § 155 Abs. 3 Satz 1 AktG.

Während in Deutschland ein Beibehaltungswahlrecht für den einmal gewählten Wert besteht, $m u \beta$ dieser in den USA fortgeführt werden. 
Weder in den USA noch in Großbritannien ist jedoch ein niedrigerer Wert, der sich aus Wertschwankungen in der Zukunft ergibt ( $\$ 155$ Abs.3 Nr .1 AktG) bzw. der aus dem Zweck der Steuern vom Einkommen oder vom Ertrag resultiert ( 155 Abs. 3 Nr. 2 AktG), zulässig.

Wesentliche und für den externen Bilanzanalytiker abschätzbare Unterschiede bestehen bei den Verfahren der Gruppenbewertung (Sammelbewertung). In Deutschland wird hauptsächlich $\mathrm{zu}$ Durchschnittskosten bewertet, in den USA verwendeten $70 \%$ der untersuchten Unternehmen zumindest für Teile des Vorratsvermögens die LIFO-, im übrigen die FIFOMethode $^{36}$. Die britischen Unternehmen bewerten typischerweise nach FIFO ${ }^{37}$.

Die US-amerikanischen Unternehmen erwähnen den Unterschiedsbetrag zwischen LIFOund FIFO-Bewertung in einer Fußnote. Diese Differenz beträgt für das Gesamtsample 1980 $5,3 \%$ der Bilanzsumme.

Eine Besonderheit für britische Unternehmen stellt der "stock appreciation relief" dar. Die steuerliche Bewertung der Vorräte wurde 1974 in Großbritannien durch die Einführung des "stock appreciation relief" geändert, da etwa $50 \%$ der Unternehmensgewinne aus inflationsbedingten Höherbewertungen des Vorratsvermögens resultierten (Scheingewinne) ${ }^{38}$. Seither wurden verschiedene Faustregeln und Indizes entwickelt, um die Steuerbilanz zu deflationieren, indem eine ,,angemessene" Entwicklung des Vorratsvermögens simuliert wird ${ }^{39}$.

Die Deflationierung in der Steuerbilanz führt zu einem niedrigeren Gewinn und damit zu einer geringeren Steuerlast als bei einer Bewertung ohne Inflationsbereinigung. In der Handelsbilanz erfolgt eine solche Deflationierung nicht. Der Differenzbetrag in den Gewinnen wird als "stock appreciation relief" bezeichnet.

Die hieraus resultierende Steuerstundung wird passivisch als eine Unterposition in die "deferred taxes" eingestellt.

Die britischen Unternehmen gehen weitgehend davon aus, daß diese Steuerstundung in Zukunft nicht aufzulösen ist. Damit wird sie ein eigenkapitalbildendes Steuergeschenk und nicht bloß ein Bilanzierungsunterschied zwischen Steuer- und Handelsbilanz. Immer mehr britische Unternehmen gehen dazu über, diese Bestandteile der "deferred taxes" in die Position „,potentielle deferred taxes" zu übernehmen. Um einen ähnlichen eigenkapitalbildenden Effekt bei den deutschen Unternehmen zu erzielen, müßte man die Gewinnsteuersätze senken.

\section{Sonstiges Umlaufvermögen}

Beim sonstigen Umlaufvermögen handelt es sich im wesentlichen um die drei Positionen Kasse und Bankguthaben (in allen Ländern zum Nennwert zu bilanzieren), die Wertpapiere des Umlaufvermögens sowie die Forderungen und Besitzwechsel.

Der Anteil an den Aktiva beträgt in Deutschland 34,2 \% (USA $29 \%$; GB 27,4 \%). Bei den Wertpapieren des Umlaufvermögens gilt in Deutschland das strenge und in den USA und Großbritannien eine Art gemildertes Niederstwertprinzip, da eine Wertberichtigung in diesen Ländern erst dann vorzunehmen ist, wenn für das gesamte Wertpapier-Portefeuille und somit nicht nur für das einzelne Wertpapier ein dauernder Wertverlust anzunehmen ist ${ }^{40}$.

Ein weiterer wichtiger Unterschied ist die Saldierung kurzfristiger Vermögenswerte mit kurzfristigen Verbindlichkeiten in Großbritannien, was zu einer erheblichen Bilanzverkürzung führt und damit eine höhere „technische" Eigenkapitalquote bewirkt.

\section{Passivseite}

Bei den Eigenkapitalpositionen sind in den betrachteten Ländern nur die Darstellungsformen verschieden ${ }^{41}$. Jedoch ergeben sich bei dem Fremdkapital einige materielle Unterschiede. 
So besteht z.B in Deutschland ein Passivierungswahlrecht für Pensionsrückstellungen, in den USA und Großbritannien erfolgt ein solcher Ausweis in der Regel nicht, da der Pensionsaufwand in den anglo-amerikanischen Jahresabschlüssen als Aufwand der Periode berechnet und meist unmittelbar an unabhängige Pensionsfonds überwiesen wird. Die unterschiedliche Behandlung der Pensionsrückstellungen erscheint beträchtlich, denn immerhin machen die Pensionsrückstellungen 10,4\% der Bilanzsumme bei den untersuchten deutschen Unternehmen aus ${ }^{42}$.

Die Passivierungsmöglichkeiten für sonstige Rückstellungen werden in Großbritannien und den USA restriktiver als in deutschen Jahresabschlüssen behandelt. In Deutschland besteht im Gegensatz zu Großbritannien und den USA die Tendenz zur Bildung von Maximalrückstellungen ${ }^{43}$. Die sonstigen Rückstellungen betragen bei den untersuchten Unternehmen in Deutschland $11 \%$ der Bilanzsumme (GB 0,4 \%; USA 5,5\%).

Lang- und kurzfristige Verbindlichkeiten werden in allen Ländern mit dem Rückzahlungsbetrag angesetzt.

\section{Berechnung der Eigenkapitalquoten}

\section{Ausgewiesenes Eigenkapital}

Bei den untersuchten Bilanzen handelt es sich zum großen Teil um Konzernabschlüsse und bei den Daten der Bonner Stichprobe um Einzelabschlüsse. Es wird daher von folgender Definition für das Eigenkapital ausgegangen ${ }^{44}$ :

Grundkapital

+ Rücklagen

+ Bilanzgewinn / - Bilanzverlust

$+0,5$ x Sonderposten mit Rücklagenanteil

+ Ausgleichsposten für Anteile im Fremdbesitz

+ Passiver Konsolidierungsausgleichsposten

- Ausstehende Einlagen auf das Grundkapital

- Eigene Aktien

= Eigenkapital $(\mathrm{EK} 1)$

Nach der „Konzeption der wirtschaftlichen Einheit“445 des Konzerns wird der „Ausgleichsposten für Anteile im Fremdbesitz" trotz seiner mißverständlichen Bezeichnung zum Eigenkapital gerechnet, da auch die Minderheitsaktionäre als Anteilseigner an Erfolg und Risiko des Konzerns teilhaben, also keine dem Konzern fremde Gläubiger sind ${ }^{46}$. Die USamerikanische und die britische Rechnungslegung folgt noch konsequenter der Einheitstheorie $^{47}$.

Der „Passive Konsolidierungsausgleichsposten" entsteht, wenn der Anteil an Kapital und Rücklagen der in den Konzernabschluß einbezogenen Untergesellschaften größer als der jeweilige Beteiligungsbuchwert ist, und hat daher grundsätzlich Rücklagencharakter ${ }^{48}$.

„Eigene Aktien" haben (wenn sie nicht nur kurzfristig zur Weiterveräußerung gehalten werden) den Charakter von Korrekturposten zu den übrigen Eigenkapitalpositionen “49. Im Rahmen der Eigenkapitalanalyse sind sie daher vom Eigenkapital abzuziehen. In den USA und in Großbritannien sind eigene Aktien prinzipiell vom ausgewiesenen Grundkapital abzusetzen.

Der Rücklagenanteil des „Sonderposten mit Rücklagenanteil" wird allgemein mit $50 \%$ angesetzt ${ }^{50}$.

Das Gesamtkapital der untersuchten Unternehmen wird folgendermaßen ermittelt ${ }^{51}$ : 
Bilanzsumme

- Ausstehende Einlagen auf das Grundkapital

- Eigene Aktien

- Bilanzverlust

- Wertberichtigungen

$=$ Gesamtkapital (GK1)

Aus der Division von Eigenkapital (EK1) durch Gesamtkapital (GK1) ergibt sich die erste Eigenkapitalquote (EQ1) die als Grundlage für die verschiedenen Korrekturen dienen soll:

$$
\mathrm{EQ} 1=\frac{\mathrm{EK} 1}{\mathrm{GK} 1}
$$

Auf dieser Basis ergeben sich für die drei Länder die folgenden Eigenkapitalquoten ${ }^{52}$ :

Tabelle 7

\begin{tabular}{lllll}
\hline & $\begin{array}{l}\text { Deutschland } \\
\text { Roniter } \\
\text { Stichprobe }\end{array}$ & $\begin{array}{l}\text { Deurschiand } \\
\text { Sample }\end{array}$ & GroBbritaniten & USA \\
\hline 1979 & $33,1 \%$ & $26,7 \%$ & $43,3 \%$ & $46,8 \%$ \\
1980 & $32,7 \%$ & $26,1 \%$ & $44,2 \%$ & $46,6 \%$ \\
\hline
\end{tabular}

2. Einflu $\beta$ des Immateriellen Anlagevermögens, des "share of retained earnings" und der Neubewertungsrücklage

Um die Vergleichbarkeit der britischen bzw. US-amerikanischen Jahresabschlüsse mit den deutschen Bilanzen herbeizuführen, wurden in einem ersten Schritt die Eigenkapitalquoten um das Immaterielle Anlagevermögen (IAV), den sich aus der Equity-Methode ergebenden "share of retained earnings of associated companies" (SRE) und die Neubewertungsrücklage (NEU) korrigiert $^{53}$. Da für die USA der SRE nicht offengelegt ist, konnte zunächst nur eine Bandbreite für die EK-Quote ermittelt werden (in Tabelle 8 Wert in Klammern). In einem weiteren Simulationslauf wurde untersucht, wie hoch die Eigenkapitalquote wäre, wenn der gleiche Anteil des "share of retained earnings of associated companies" an dem Finanzanlagevermögen unterstellt würde wie in Großbritannien ${ }^{54}$.

Tabelte 8

\begin{tabular}{llllll}
\hline & $\begin{array}{l}\text { Deutschland } \\
\text { bonner } \\
\text { Szichprabe }\end{array}$ & $\begin{array}{l}\text { Deutschland } \\
\text { Sample }\end{array}$ & Gro\&britantrien & [USR] & USA \\
\hline 1979 & $32,5 \%$ & $26,6 \%$ & $38,8 \%$ & $(45,2 \%)$ & $43,4 \%$ \\
1980 & $32,1 \%$ & $25,8 \%$ & $40,1 \%$ & $(45,1 \%)$ & $43,0 \%$ \\
\hline
\end{tabular}

\section{Berücksichtigung der "deferred taxes "}

a) Bilanziell ausgewiesene deferred taxes $(D T A)^{55}$

Da in Deutschland, wie empirische Untersuchungen zeigen ${ }^{56}$, hauptsächlich degressiv abgeschrieben wird, verkürzt sich die Bilanzsumme um $\triangle \mathrm{SAV}=\left(\mathrm{SAV}_{\mathrm{L}}-\mathrm{SAV}_{\mathrm{D}}\right)$ gegenüber einer linearen Abschreibung. Bei einem Steuersatz von T entspricht dem auf der Passivseite 
eine Verkürzung um die Steuerschuld von $\mathrm{T} \times \Delta \mathrm{SAV}$, und damit vermindert sich das Eigenkapital (durch Bildung Stiller Reserven) um $\triangle \mathrm{EK}=(1-\mathrm{T}) \mathrm{x} \Delta \mathrm{SAV}$.

In Großbritannien und den USA bleibt auf der Aktivseite, da in diesen Ländern in der Handelsbilanz meist linear abgeschrieben wird, $\mathrm{SAV}_{\mathrm{L}}$ stehen. Auf der Passivseite wird DTA = $\mathrm{T} x \Delta \mathrm{SAV}$ als "deferred taxes" passiviert ${ }^{57}$. Das Eigenkapital bleibt um $\triangle \mathrm{EK}=(1-\mathrm{T}) \mathrm{x} \Delta \mathrm{SAV}$ höher als es sich nach der in Deutschland üblichen Bilanzierung ergeben würde.

Die Korrektur der Eigenkapitalquote EQ2 kann deshalb in zwei Schritten vorgenommen werden:

1. Reduzierung des Eigenkapitals der britischen und US-amerikanischen Unternehmen um $\Delta \mathrm{EK}=(1-\mathrm{T}) \mathrm{x} \Delta \mathrm{SAV}$.

Damit ergibt sich als neues Eigenkapital ${ }^{58}$ :

$\mathrm{EK} 3=\mathrm{EK} 2-(1-\mathrm{T}) \mathrm{x} \triangle \mathrm{SAV}$.

2. Reduzierung der "deferred taxes" um den auf Abschreibungsunterschieden beruhenden Anteil DTA $=$ T x $\triangle \mathrm{SAV}$.

Hieraus ergibt sich das neue Gesamtkapital ${ }^{59}$ :

GK3 $=$ GK2 $-\triangle \mathrm{EK}-\mathrm{DTA}=\mathrm{GK} 2-\triangle \mathrm{SAV}$.

Da aus den untersuchten Bilanzen DTA, EK2, GK2 und T bekannt sind und außerdem gilt, daß SAV = 1/T x DTA, läßt sich die neue Eigenkapitalquote (EQ3) folgendermaßen ermitteln:

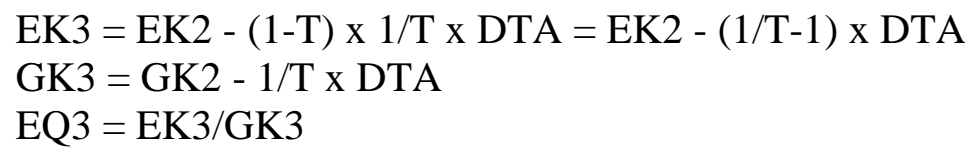

$\mathrm{Zu}$ den Auswirkungen der „bilanziell ausgewiesenen deferred taxes" auf das Sachanlagevermögen vergleiche Bild 4.

Für die Unternehmen des betrachteten Samples ergeben sich unter Berücksichtigung der „,bilanziell ausgewiesenen deferred taxes" folgende neue Eigenkapitalquoten:

Tabelies

\begin{tabular}{lllll}
\hline & $\begin{array}{l}\text { Deursehtand } \\
\text { Botner } \\
\text { Seichprobe }\end{array}$ & $\begin{array}{l}\text { Deurschland } \\
\text { Sample }\end{array}$ & Gro\&britarnien & USA \\
\hline 1979 & $32,5 \%$ & $26,6 \%$ & $37,8 \%$ & $42,5 \%$ \\
1980 & $32,1 \%$ & $25,8 \%$ & $39,5 \%$ & $42,0 \%$ \\
\hline
\end{tabular}


Bild 4: Vergleich der US-aıneriksnischen usd der bribschen Bilanz nach der Korreksur des Sacharlagevermögens um "deferred taxes" und Neubewertung

AMERIKANISCHE BILANZ

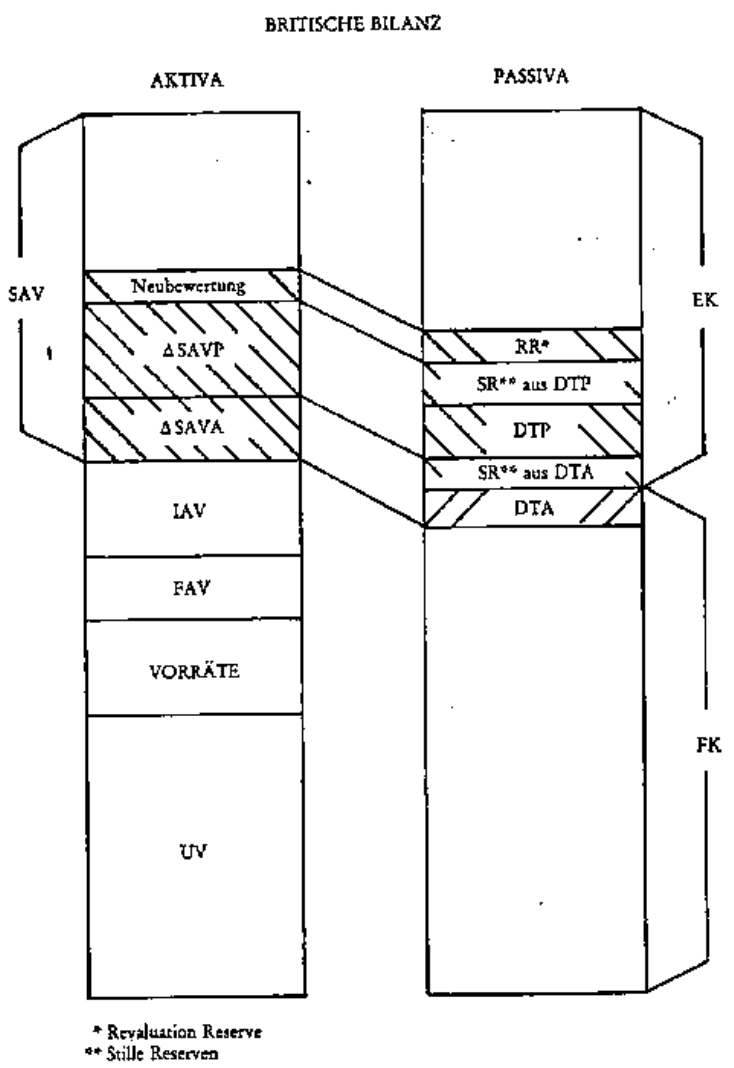

b) Potentielle deferred taxes (DTP)

Während bei den „bilanziell ausgewiesenen deferred taxes" (DTA) nur ein Teil der Abschreibungsdifferenz, nämlich (1/T-1) x DTA eigenkapitalbildend ist, werden in Großbritannien außerdem die vollen ,,potentiellen deferred taxes" als Gewinn behandelt ${ }^{60}$. Es gilt hier:

$\mathrm{DTP}=\mathrm{T} \times \triangle \mathrm{SAVP}^{61}$ und umgekehrt $: \triangle \mathrm{SAVP}=1 / \mathrm{T} \times \mathrm{DTP}$

EK4 $=$ EK3 $-(1 / \mathrm{T}-1)$ DTP - DTP $=$ EK3 $-1 / \mathrm{T}$ x DTP

„Stille Reserve"nach deutscher Bilanzierung

GK4 $=$ EK3 $-\triangle \mathrm{SAVP}=\mathrm{GK} 3-1 / \mathrm{T} \times \mathrm{DTP}$

$\mathrm{EQ} 4=\mathrm{EK} 4 / \mathrm{GK} 4$

$\mathrm{Zu}$ den Auswirkungen der ,potentiellen deferred taxes" auf das Sachanlagevermögen vergleiche Bild 4.

Für die Unternehmen des betrachteten Samples ergeben sich die folgenden neuen Eigenkapitalquoten: 


\begin{tabular}{lllll}
\hline & $\begin{array}{l}\text { Deutschland } \\
\text { Bonther } \\
\text { Seichprobe }\end{array}$ & $\begin{array}{l}\text { Deutschland } \\
\text { Sample }\end{array}$ & Großbrizanties & USA \\
\hline 1979 & $32,5 \%$ & & & \\
1980 & $32,1 \%$ & $26,6 \%$ & $31,7 \%$ & $42,5 \%$ \\
\hline
\end{tabular}

\section{c) Beispiel}

Das folgende Beispiel soll die Ermittlung der Eigenkapitaldifferenzen aus den unterschiedlichen Abschreibungsmethoden zwischen britischen und deutschen Unternehmen verdeutlichen.

Gegeben sei die in Bild 5 gezeigte britische Bilanz, wobei von einer linearen Abschreibung ausgegangen wurde. Die Differenz zwischen linearer und der in Deutschland üblichen degressiven Abschreibung soll 200 betragen. Dabei wird unterstellt, daß die ,potentiellen deferred taxes" (DTP) 78 betragen.

\section{Bilds}

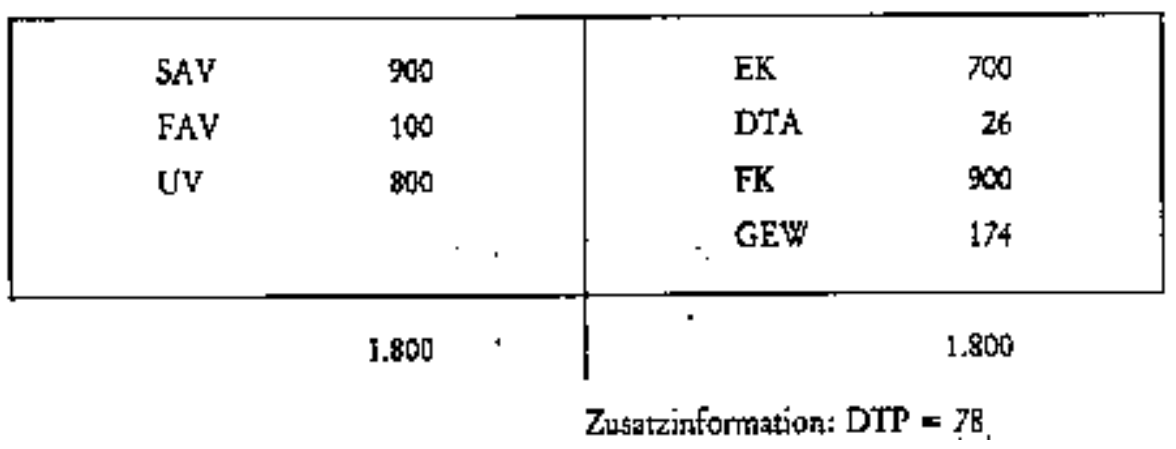

Die Eliminierung der 200, die in Deutschland $\mathrm{zu}$ einer Verringerung des Sachanlagevermögens führen würden, kann aus den britischen Bilanzen wie folgt vorgenommen werden:

1. Schritt: Ermittlung der Unterschiede im Sachanlagevermögen ( $\triangle$ SAVA) aus den „bilanziell ausgewiesenen deferred taxes“ (DTA). (Annahme: $\mathrm{T}=0,52)$ :

$\triangle \mathrm{SAVA}=\mathrm{DTA} / \mathrm{T}$

$\triangle \mathrm{SAVA}=26 / 0,52=50$

2. Schritt: Ermittlung der Unterschiede im Sachanlagevermögen $(\triangle \mathrm{SAVP})$ aus den „potentiellen deferred taxes“(DTP):

$\triangle \mathrm{SAVP}=\mathrm{DTP} / \mathrm{T}$

$\triangle \mathrm{SAVP}=78 / 0,52=150$

3. Schritt: Ermittlung des korrigierten Sachanlagevermögens (SAVN):

$\triangle \mathrm{SAV}=\triangle \mathrm{SAVA}+\triangle \mathrm{SAVP}$

$\triangle \mathrm{SAV}=200$

$\mathrm{SAVN}=\mathrm{SAV}-\triangle \mathrm{SAV}$

SAV $=900-200=700$

4. Schritt: Korrektur des Gewinns um die „bilanziell ausgewiesene deferred taxes“ ( $\triangle$ GEWA):

$\triangle \mathrm{GEWA}=(\triangle \mathrm{SAVP}-\mathrm{DTP})+\mathrm{DTP}$

$\triangle \mathrm{GEWA}=50-26=24$ 
5. Schritt: Korrektur des Gewinns um die ,potentiellen deferred taxes“( $\triangle$ GEWP):

$\triangle \mathrm{GEWP}=(\triangle \mathrm{SAVP}-\mathrm{DTP})+\mathrm{DTP}$

$\triangle$ GEWP $=150$

6. Schritt: Korrektur des Gesamtgewinns (GEWN):

GEWN $=$ GEW $-\triangle$ GEWA $-\triangle$ GEWP

GEWN $=174-24-150$

GEWN $=0$

7. Schritt: Darstellung der um die Differenz zwischen linearer und degressiver Abschreibung korrigieren britischen Bilanz:

Bild 6

\begin{tabular}{|ll|lr|}
\hline SAVN & 700 & EK & 700 \\
FAV & 100 & DTA & 0 \\
UV & 800 & FX & 900 \\
& & GEWN & 0 \\
\hline & 1,600 & & 1.600 \\
\hline
\end{tabular}

Diese Bilanz entspricht der deutschen Bilanzierung bei degressiver Abschreibung.

In dem Beispiel ist damit der ursprüngliche britische Eigenkapitalanteil in Höhe von $48,56 \%(874 / 1800)$ auf 43,75\% (700/1600) gesunken.

Es muß jedoch bei der Behandlung der "deferred taxes" berücksichtigt werden, daß alle steuerlichen Abschreibungen, also auch Sonderabschreibungen nach deutschem Recht, in diese Position mit ihrer steuerlichen Wirkung eingerechnet werden. Wie Tabelle 6 zeigt, sind in Großbritannien jedoch u.U. $100 \%$ ige Abschreibungen zulässig ${ }^{62}$. Es könnte somit mitunter auch vorkommen, daß eine $\mathrm{zu}$ pessimistische Abschätzung der Verringerung des Sachanlagevermögens erfolgt.

Jedoch müssen dem die Abschreibungen in Deutschland, wie z.B. für geringwertige Wirtschaftsgüter entgegengerechnet werden.

\section{Einflu $\beta$ der FIFO- bzw. LIFO-Bewertung der Vorräte auf die Eigenkapitalquote}

a) LIFO-Methode in den USA

Wie bereits in Kapitel III 4 dargestellt, wäre bei den untersuchten US-amerikanischen Unternehmen die Bilanzsumme um 5,3 \% größer geworden, wenn sie statt der LIFO- die FIFOMethode angewendet hätten.

Da die britischen Unternehmen nach der FIFO-Methode bilanzieren, wird zunächst eine Angleichung der US-amerikanischen Vorratsbewertung an die britische vorgenommen. In Abschnitt IV 4 b) wird dann eine Angleichung der FIFO-Methode an die in Deutschland übliche Durchschnittsmethode durchgeführt. Die amerikanischen Unternehmen veröffentlichen den Unterschiedsbetrag zwischen den nach LIFO bilanzierten Vorräten zu einer FIFO-Bewertung der Vorräte $(\triangle \mathrm{VOR}=\mathrm{VORF}-\mathrm{VORL})$ in einer Fußnote. 
Unter der Annahme einer Steuerrate (T) von $48 \%$, einer vollen Thesaurierung des Eigenkapitalzuwachses und einer Passivierung der Steuerverbindlichkeiten ergibt sich für die US-amerikanischen Unternehmen:

$$
\begin{aligned}
& \mathrm{GK} 5=\mathrm{GK} 4+\underbrace{(1-\mathrm{T}) \times \Delta \mathrm{VOR}}_{\Delta \text { Eigenkapital }}+\underbrace{\mathrm{T} \times \Delta \mathrm{VOR}}=\mathrm{GK} 4+\Delta \mathrm{VOR} \\
& \mathrm{EK} 5=\mathrm{EK} 4+(1-\mathrm{T}) \mathrm{x} \Delta \mathrm{VOR} \\
& \mathrm{EQ} 5=\frac{\mathrm{EK} 4+0,52 \mathrm{x} \Delta \mathrm{VOR}}{\mathrm{GK} 4+\Delta \mathrm{VOR}}
\end{aligned}
$$

Wie aus Tabelle 11 ersichtlich, steigt der Eigenkapitalanteil der betrachteten USamerikanischen Unternehmen durch die Angleichung an die britische Bilanzierung leicht an.

Tabelte 11

\begin{tabular}{lcc} 
& $\mathrm{EQ}$ & $\mathrm{EQj}$ \\
\hline $197 \%$ & $42,5 \%$ & $42,7 \%$ \\
1980 & $42,0 \%$ & $42,4 \%$ \\
\hline
\end{tabular}

\section{b) Die Umrechnung der FIFO- auf die Durchschnittskostenmethode}

Nach der FIFO-Methode wird unterstellt, daß die zuerst beschafften Bestände auch zuerst verbraucht und damit die Lagerbestände mit den Einstandspreisen der letzten Lieferungen (VORF) bewertet werden. Bei einer großen Umschlagshäufigkeit entspricht dieser Wert dem Preis gegen Ende der betrachteten Periode.

Nach der Durchschnittskostenmethode (VORD) wird aus den Anfangsbeständen und den Zugängen der Periode ein gewogener Durchschnittspreis gebildet; d.h. der Preis liegt damit zwischen dem Anfangs- $\left(\mathrm{P}_{\mathrm{A}}\right)$ und Endpreis $\left(\mathrm{P}_{\mathrm{E}}\right)$ der Periode. Für eine grobe Umrechnung gilt dann $^{63}$ :

$$
\mathrm{VORD}=\mathrm{VORF} \times \frac{(\mathrm{PE}+\mathrm{PA})}{2 \times \mathrm{PE}}
$$

Basis der Preisberechnung sind die Indizes der Großhandels- bzw. gewerblichen Erzeugerpreise für die USA bzw. Großbritannien ${ }^{64}$.

Für das Vorratsvermögen ergeben sich folgende Gleichungen:

$$
\begin{aligned}
& \text { USA (1979): VORD }=0,944 \text { VORF } \\
& \text { USA (1980): VORD }=0,038 \text { VORF } \\
& \text { GB (1979): VORD }=0,946 \text { VORF } \\
& \text { GB (1980): VORD }=0,93 \text { VORF }
\end{aligned}
$$

Die Eigenkapitalquoten der USA und Großbritanniens verändern sich aus diesen Umrechnungen auf die in Deutschland übliche Durchschnittsmethode nur geringfügig (vgl. Tabelle 12). 
Tabelle 12

\begin{tabular}{lcccc}
\hline \multicolumn{2}{c}{ Grobbritannien } & \multicolumn{2}{c}{ USA } \\
\hline & EQS & EQ6 & EQ5 & EQ6 \\
\hline 1979 & $31,7 \%$ & $31,3 \%$ & $42,7 \%$ & $42,6 \%$ \\
1900 & $32,7 \%$ & $32,2 \%$ & $42,4 \%$ & $42,3 \%$ \\
\hline
\end{tabular}

\section{Berücksichtigung der Pensionsrückstellungen}

In den USA und Großbritannien werden die Aufwendungen für die betriebliche Altersversorgung in der Regel an unabhängige "pension funds" abgeführt, in Deutschland werden meist Pensionsrückstellungen gebildet. Im zugrunde liegenden Sample betrugen sie 1980 10,4 \% der Passivseite.

Die Frage, ob Teile der Pensionsrückstellungen auch Eigenkapitalcharakter haben, ist in der Literatur umstritten. Die Bundesbank behandelt sie in ihrer Bilanzstatistik als Fremdmittel, obwohl sie ,eigenerwirtschaftete und in gewisser Weise billige, auch nicht jederzeit fällige Finanzierungsmittel ${ }^{\text {‘65 }}$ darstellen, da sie (z.B. im Konkursfall) keine Haftungsfunktion besitzen ${ }^{66}$.

Interessant ist jedoch die Überlegung, wie sich die Eigenkapitalquote verändern würde, wenn die deutschen Unternehmen wie die britischen und US-amerikanischen Unternehmen den Pensionsaufwand nicht passivieren, sondern abführen müßten.

Es wurden drei Alternativen durchgerechnet:

1. Unterstellt man, daß in deutschen Unternehmen keine Pensionsrückstellungen gebildet würden und die jährlichen Zuführungen zur Pensionsrückstellung in unabhängige Pensionsfonds überführt würden, dann käme dies einer Bilanzverkürzung gleich. Das würde implizieren, daß die Aktiva in gleicher Weise reduziert werden, ohne daß dies einen Einfluß auf die Ertrags- und damit die Eigenkapitalsituation hätte. Diese Annahme erscheint zumindest problematisch.

Die Eigenkapitalquote erhöht sich dann 1979 von $26,7 \%$ auf $30,5 \%$ und 1980 von $25,8 \%$ auf $29,1 \%$.

2. Geht man davon aus, daß die Bilanzsumme konstant bleibt und das Unternehmen sich bemüht, den Mittelabfluß durch Zuführung von Eigen- und Fremdmitteln im Verhältnis der vorherigen Eigenkapitalquote zu ersetzen, so gilt:

$\mathrm{EQ7}=\frac{\mathrm{EK} 2+\mathrm{EQ} 2 \times \mathrm{PRSt}}{\mathrm{GK} 2}$

Die Eigenkapitalquote der deutschen Unternehmen im betrachteten Sample erhöht sich 1979 von $26,7 \%$ auf $29,8 \%$ und 1980 von $25,8 \%$ auf $28,8 \%$.

3. Die privaten amerikanischen "pension funds" investieren einen großen Teil ihrer Mittel in Aktien; 1973 waren es 66,9 \% ${ }^{67}$, für 1980 wird der Aktienanteil an den Vermögenswerten der "private pension funds" auf $50 \%$ geschätzt ${ }^{68}$. Insgesamt hielten die (private and governmental) "pension funds" 1980 nicht weniger als $15 \%$ des Marktwertes aller Aktien in den USA, so daß sie schon als die „neuen Eigentümer der amerikanischen Großunternehmen" bezeichnet wurden ${ }^{69}$. Unterstellt man, daß ,deutsche Pensionsfonds" genauso handelten, so ergäbe sich bei der vorsichtigen Annahme, daß der Mittelrückfluß zu $50 \%$ ins Eigenkapital und zu $50 \%$ in die Fremdmittel ginge, eine Erhöhung der Eigenkapitalquote für 1979 von 26,7 \% auf 31,8 \% und für 1980 von $25,8 \%$ auf $31,1 \%$.

Tabelle 13 faßt die Ergebnisse der Untersuchung zusammen. 
Aus ihr wird deutlich, daß die Eigenkapitalanteile bei deutschen und britischen Unternehmen sich nur noch geringfügig unterscheiden. Damit kann bei aller gebotenen Vorsicht kaum von einer besseren Eigenkapitalausstattung der britischen Unternehmen gesprochen werden.

Tabelle 13

\begin{tabular}{|c|c|c|c|c|c|c|}
\hline & \multicolumn{2}{|c|}{$\begin{array}{l}\text { Deuschland } \\
\text { Bouner } \\
\text { Stichprobe }\end{array}$} & \multicolumn{2}{|c|}{$\begin{array}{l}\text { Deutsehtand } \\
\text { Sample }\end{array}$} & \multirow[t]{2}{*}{ Grofbritannien } & \multirow[t]{2}{*}{ USA } \\
\hline & 1 & 2 & $\mathbf{I}$ & 2 & & \\
\hline $\begin{array}{l}1979 \text { AEQ } \\
1979 \mathrm{KEQ} \\
1990 \mathrm{AEQ} \\
1990 \mathrm{KEQ}\end{array}$ & $\begin{array}{l}33,1 \% \\
32,5 \% \\
32,7 \% \\
32,1 \%\end{array}$ & $\begin{array}{l}33,1 \% \\
37,1 \% \\
32,7 \% \\
37,3 \%\end{array}$ & $\begin{array}{l}26,7 \% \\
26,6 \% \\
26,1 \% \\
25,8 \%\end{array}$ & $\begin{array}{l}26,7 \% \\
31,8 \% \\
26,1 \% \\
31,1 \%\end{array}$ & $\begin{array}{l}43,3 \% \\
31,3 \% \\
44,2 \% \\
32,2 \%\end{array}$ & $\begin{array}{l}46,8 \% \\
42,6 \% \\
46,6 \% \\
42,3 \%\end{array}$ \\
\hline $\begin{array}{l}\text { 1: Ohne B } \\
\text { 2: Min Beri } \\
\text { AEQ: Asts } \\
\text { KEQ: Kort }\end{array}$ & re Eig & $\begin{array}{l}\text { der P } \\
\text { Per Per } \\
\text { kapit }\end{array}$ & $\begin{array}{l}\text { onstick } \\
\text { srückst } \\
\text { tre }\end{array}$ & litngen & & \\
\hline
\end{tabular}

Auch der anfangs beträchtliche Unterschied zwischen deutschen und US-amerikanischen Unternehmen, der 1980 im betrachteten Sample 20,5 Prozentpunkte betrug, reduzierte sich auf 11,2 Prozentpunkte.

Die vorgelegte Untersuchung blieb weitgehend frei von Spekulationen, so daß die deutschen Unternehmen mit ihrer ,vorsichtigen" Bilanzierung eine eher etwas zu niedrige Eigenkapitalquote ausweisen. Die Einflüsse, die aus den Unterschieden zwischen dem angloamerikanischen Prinzip des "true and fair view", das leicht zu einem "window-dressing" führen kann und dem „Vorsichtsprinzip" in der deutschen Bilanzierung resultieren, können nur durch Simulationsrechnungen abgeschätzt werden. Dies gilt insbesondere für die Abschreibungen für außerplanmäßigen, technisch oder wirtschaftlich bedingten Wertverlust, für die Bewertung der Vorräte nach Voll- oder Teilkosten, für die Unterschiede in der Anwendung des Niederstwertprinzips und für die Bilanzierung der anderen Rückstellungen.

Auffallend ist beispielsweise der Unterschied in den anderen Rückstellungen zwischen den US-amerikanischen und den deutschen Unternehmen (USA 5,5\%; D $11 \%$ ). Geht man davon aus, daß die tatsächliche Risikosituation US-amerikanischer und deutscher Unternehmen im Durchschnitt nicht gravierend unterschiedlich ist, so muß der Grund für die Differenz in der unterschiedlichen bilanziellen Behandlung der Vorsorge für ungewisse Zukunftsverpflichtungen gesucht werden. Ein Indiz dafür ist die traditionell starke Betonung des Vorsichtsprinzips in Deutschland, das bei einer Risikovorsorge via Rückstellung auch zu Steuervorteilen gegenüber der Rücklage führt ${ }^{70}$, während in den USA nur bei „wahrscheinlichem" (nach Schoenfeld höchstwahrscheinlichem"71) Auftreten einer zukünftigen Verpflichtung eine Rückstellung gebildet werden darf $^{72}$; in den übrigen Fällen sind spezielle Rücklagen zu bilden und Angaben in Fußnoten zu machen ${ }^{73}$. Bei einer Differenz von 5,5 Prozentpunkten und einem US-amerikanischen Steuersatz von $48 \%$ dürfte sich hier eine Angleichung der Eigenkapitalquoten von zwei bis drei Prozentpunkten ergeben.

Interessant erscheint die bilanzielle Behandlung der "deferred taxes" in Großbritannien. „Stille Reserven", die in Deutschland durch degressive Abschreibungen gebildet werden können, gehen über die Steuereffekte der "deferred taxes" in Großbritannien in den Gewinn ein. Damit werden auch die britischen ausgewiesenen Eigenkapital- und Umsatzrentabilitäten im Verhältnis zu den deutschen beträchtlich zu hoch ausgewiesen und bedürfen der Korrektur.

Der hier dargestellte Bilanzvergleich macht deutlich, daß die in der Öffentlichkeit immer wieder geltend gemachten Forderungen nach Verbesserung der Eigenkapitalanteile deutscher Unternehmen im Vergleich zu ausländischen nicht unumstritten bleiben dürfen, solange eine gemeinsame Vergleichsbasis nicht vorhanden ist. Hier wurde deutlich, daß die deutschen Unternehmen einen solchen Vergleich mit britischen Unternehmen nicht zu scheuen brauchen. Auch die Unterschiede zu den US-amerikanischen Unternehmen sind weit weniger dramatisch 
(7-8 \% unter Berücksichtigung der anderen Rückstellungen) als dies vielfach dargestellt wird. Die Daten der Bonner Stichprobe führen unter Berücksichtigung der anderen Rückstellungen sogar zu einer gleichen Eigenkapitalausstattung wie bei US-amerikanischen Unternehmen ${ }^{74}$. Es kann also nicht davon ausgegangen werden, daß „die länderweisen Unterschiede z.T. so spektakulär (sind), daß begrenzungs- und bewertungsrelevante Differenzen ihre Bedeutung mehr oder weniger einbüßen"75. Inwieweit eine Vergleichbarkeit mit französischen und japanischen Unternehmen hergestellt werden kann, soll in einer weiteren Untersuchung festgestellt werden.

1. ALBACH, Steigende Verschuldung und sinkende Eigenkapitalrentabilität deutscher Unternehmen, Die AG 1979, 121 ff; BOFINGER, Nur eine Legende? Wirtschaftswoche Nr. 27, 1983, 48 ff; Monatsberichte der Deutschen Bundesbank, November 1983, Ertragslage und Finanzierungsverhältnisse der Unternehmen im Jahre 1982, 14 ff; DRUKARCZYK, Korrekturen in der Kapitalstruktur und Eigentümerinteressen, Regensburger Diskussionsbeiträge zur Wirtschaftswissenschaft, 1984; FISCHER, Die steuerliche Belastung der Unternehmensfinanzierung im internationalen Vergleich und ihr Einfluß auf die Wettbewerbsfähigkeit deutscher Unternehmen, DB 1984, 357ff ; FRITSCH, Die Eigenkapitallücke in der Bundesrepublik, 1981; GRUHLER, Unternehmensrenditen und Eigenkapitalausstattung im internationalen Vergleich, Die AG 1984, 100ff; Sachverständigenrat, Jahresgutachten 1982/83, BT-Drucks. 9/2118, Tz. 301 ff; SCHLESINGER, Finanzielle Schwächepunkte der deutschen Wirtschaft, Kredit und Kapital 1982, 473 ff; DERS., Unternehmensfinanzierung und Wettbewerbsfähigkeit, ZfbF 1984, 6 ff.

2. Verneint wird die Existenz einer "Eigenkapitallücke" von: FLASSBECK/KOLL, Kapital und Rendite, Wirtschaftswoche Nr. 21, 1983, 72 ff; Rheinisch-Westfälisches Insitut für Wirtschaftsforschung, Analyse der strukturellen Entwicklung der deutschen Wirtschaft (Strukturbericht 1983), Bd. I, 1983, S. 142 ff; THORMÄHLEN/MICHALK, Leiden die deutschen Unternehmen an mangelnder Eigenkapitalausstattung? Wirtschaftsdienst 1983, $87 \mathrm{ff}$.

3. Vgl. FISCHER, DB 1984, 357ff; GRUHLER, Die AG 1984, 100, 104; SCHLESINGER, ZfbF 1984, 6 ff.

4. Vgl. die in Fn. 1 genannten Autoren.

5. BUCH, Die Pensionsrückstellungen und die stillen Reserven vermögen "echtes" Eigenkapital nicht zu ersetzen, Handelsblatt vom 13./14.1.1984, 13; FISCHER, DB 1984, 357 f; FRITSCH, aaO (Fn. 1), S. 27f; GRUHLER, Unternehmensrenditen und Eigenkapitalausstattungen im internationalen Vergleich, iwtrends 4 (1983), 55, 62; DERS., Die AG 1984, $100 \mathrm{ff}$.

6. GOERDELER, Bilanzierung und Publizität im internationalen Vergleich, ZfbF 1982, 235, 239 ff; GRAM, The Impact of International Accounting Differences from a Security-Analysis Perspective: Some European Evidente, Journal of Accounting Research, Spring 1980, 64 ff; zu den unterschiedlichen Zielsetzungen von Jahresabschlüssen in den verschiedenen Ländern vgl. PERLITZ, Internationale Zusammenarbeit auf dem Gebiet der Bilanztheorie, ZfB, Ergänzungsheft 1, 1981, 147ff.

7. Dieses Problem wird ausführlich erörtert bei GONEDES/DOPUCH, Economic Analyses and Accounting Techniques: Perspective and Proposals, Journal of Accounting Research, Autumn 1979, 334 ff; ein interessanter Ansatz zur Modellanalyse internationaler Rechnungslegung findet sich bei MACHARZINA, Grenzen einer internationalen Vereinheitlichung der Rechnungslegung, in: Rühli/Thommen (Hrsg.), Unternehmungsführung aus finanz- und bankwirtschaftlicher Sicht, 1981, S. $365,370 \mathrm{ff}$.

8. Vgl. Fn. 10-12.

9. GRUHLER, Die AG 1984, 100.

10. Vgl. BUCH, Handelsblatt vom 13./14.1.1984, 13; GRUHLER, iw-trends 4 (1983), 55, $61 \mathrm{f}$.

11. GRUHLER, Die AG 1984, 100, 103; SCHLESINGER, ZfbF 1984, 6, 14 f.

12. FISCHER, DB 1984, 357, 364; WEITKEMPER, Diskussionsbeitrag zum Thema „Die Eigenkapitalknappheit in der Wirtschaft", ZfgK 1983, 1094 ff.

13. Jahresabschlüsse der Unternehmen in der Bundesrepublik Deutschland 1965 bis 1981, Sonderdrucke der Deutschen Bundesbank, Nr. 5, 1983, S. 18.

14. GRUHLER, iw-trends 4 (1983), 55, 62 ; FRITSCH, aaO (Fn. 1), S. 28. 
15. Bei der Berechnung von Anschaffungs- und Herstellungskosten ergeben sich für das Anlagevermögen kaum Unterschiede in den drei betrachteten Ländern.

16. Vgl. KOLL, Rentabilität und Inflation, 1979 und SCHILDBACH, Geldentwertung und Bilanz, 1979, m.w.N.

17. Etwa in der Preissteigerungsrücklage gem. § 74 EStDV.

18. Vgl. Financial Accounting Standards Board Statement No. 33: Financial reporting and changing prices (1979); siehe auch JUNG, US-amerikanische und deutsche Rechnungslegung, 1979, S. 233 ff.

19. Vgl. Statements of Standard Accounting Practice No. 16: Current Cost accounting (1980); sowie LEE, G. A., Modern Financial Accounting, 3. Aufl., Wokingham 1983, S. 290 ff.

20. Die britischen Unternehmen erläutern in einer Fußnote zum Anlagevermögen, welcher Teil des Bruttoanlagevermögens zu Anschaffungskosten und welcher zu Wiederbeschaffungspreisen bewertet wird. Aus den ausgewiesenen Neubewertungsrücklagen und dem neubewerteten Anlagevermögen läßt sich ein Quotient bilden, der für die Gesamtheit der untersuchten Unternehmen als Schätzgröße des im Wert der neubewerteten Anlagegüter enthaltenen Rücklagenanteils dient. Dieser Schätzparameter wurde aus der Summe der Unternehmen, welche die volle Neubewertungsrücklage ausweisen und denen, welche nur den Zuwachs der Neubewertungsrücklage im Jahr 1980 offengelegt haben, ermittelt. Er ist daher eher zu niedrig angenommen. Durch Multiplikation des Schätzparameters mit dem Betrag des neubewerteten Anlagevermögens der Unternehmen, welche keine Neubewertungsrücklage ausgewiesen haben, wurde dann die ,versteckte“ Neubewertungsrücklage geschätzt.

21. Vgl. COENENBERG, Jahresabschluß und Jahresabschlußanalyse, 6. Aufl., 1982.

22. Siehe hierzu auch SCHMIDLE, Das Publizitätsverhalten Deutscher Aktiengesellschaften im Erläuterungsbericht gem. $§ 160$ Abs. 2 Satz 2-5 AktG, 1981, S. 50 ff.

23. Zur Bilanzierung der "deferred taxes" in Großbritannien vgl. Statement of Standard Accounting Practice No. 15: Accounting for deferred taxation (1978) ; LEE, aaO (Fn. 19), S. 241 sowie mit einfachen Beispielen LEWIS/FIRTH, Foundation in Accounting, Bd. 2, London 1977, S. 182 ff. Für die USA siehe: JUNG, aaO (Fn. 18), S. 134 ff und die ausführliche Darstellung bei KIESO/WEYGANDT, Intermediate Accounting, 4. Aufl., New York 1983, S. $886 \mathrm{ff}$.

24. Bis zum 29.7.1981 konnten maximal $25 \%$ abgeschrieben werden (vgl. § 7 Abs. 2 Satz 2 EStG).

25. Zur besseren Vergleichbarkeit wird angenommen, daß ebenfalls die geometrischdegressive Abschreibungsmethode zugrundeliegt, obwohl in den USA und Großbritannien andere degressive Abschreibungsmethoden üblich sind (eine Übersicht findet sich bei LEE, aaO [Fn .19], S. $100 \mathrm{ff}$ ).

26. Siehe Statement of Standard Accounting Practice No. 15: Accounting for deferred taxation (1978), zitiert nach: LEE, aaO (Fn. 19), S. 289.

27. Vgl. Auch: Institut für Finanzen und Steuern, Steuerrechtliche Behandlung von Investitionen in der Europäischen Gemeinschaft, Grüner Brief Nr. 190, 56 ff, insbes. 60 f.

28. Vgl. zum Immateriellen Anlagevermögen in Deutschland: MAUL, Immaterielles Anlagevermögen, Prüfung, in: Handwörterbuch der Revision, 1983, Sp. 617 ff m.w.N.

29. Ingangsetzungsaufwendungen zählen nicht zum eigentlichen Immateriellen Anlagevermögen (vgl. MAUL, aaO [Fn .28], Sp. 617, 621); bei der Aggregation zu Hauptpositionen der Bilanz werden sie aber hier erfaßt (vgl. Wirtschaftsprüfer-Handbuch 1981, S. 712 f).

30. Siehe JUNG, aaO (Fn. 18), S. 126.

31. Vgl. BUSSE VON COLBE/ORDELHEIDE, Konzernabschlüsse, 4. Aufl., 1983, S. 84 f.

32. Vgl. JUNG, aaO (Fn. 18), S. 109 ff; LEE, aaO (Fn.19) S. 383 ff.

33. Vgl. LEE, aaO (Fn. 19), S. 382.

34. Für eine detailliertere Darstellung siehe HEINEN/WIEDERMANN, Prüfung der Herstellungskosten, in: Handwörterbuch der Revision, 1983, Sp. 602 ff, insbes. Sp. 606 f; zur Bilanzierungspraxis siehe SCHMIDLE, aaO (Fn. 22), S. $90 \mathrm{ff}$.

35. Für Großbritannien siehe LEE, aaO (Fn. 19), S. 382; für die USA vgl. KIESO/WEYGANDT, aaO (Fn. 23), S. 335 ff.

36. Für ein größeres Sample von 1073 Unternehmen berichtet "Accounting Trends and Techniques" für 1981, daß 396 Unternehmen die LIFO-Methode, 382 die FIFOMethode, 238 die Durchschnittskostenmethode und 57 Unternehmen andere Methoden benutzten (zitiert nach KIESO/WEYGANDT, aaO [Fn .23], S .363). Besonders bei großen Unternehmen besteht aber eine Tendenz zur LIFO-Bilanzierung, was mit den relativ hohen fixen Kosten der LIFO-Bilanzierung zusammenhängen mag; vgl. MORSE/RICHARDSON, The LIFO/FIFO Decision, Journal of Accounting Research, Spring 1983, 106, 111.

37. Vgl. LEE, aaO (Fn. 19) S. 73.

38. Siehe BUTTERWORTHS, UK Tax Guide 1982-1983, London 1983, S. 234 f; zur Scheingewinnbesteuerung vgl. FISCHER, DB 1984, 357, $363 \mathrm{f}$.

39. Vgl. zu den Verfahren im einzelnen LEWIS/FIRTH, aaO (Fn. 23), S. 186 f; BUTTERWORTHS, aaO (Fn. 38) S. 235.

40. Vgl. MÜLLER, Praktische Bilanzierungsprobleme im Vergleich USA/Deutschland, ZfbF 1982, $249,253$. 
41. Vgl. JUNG, aa0 (Fn. 18), S. 49 f; LEE, aaO (Fn. 19) S. 220 ff.

42. Eine Untersuchung von 475 der 500 größten amerikanischen Unternehmen im Jahr 1978 ergab, daß die Pensionsaufwendungen im Schnitt 12,5\% des Gewinns vor Steuer ausmachten. Die gesamten Pensionsverbindlichkeiten (vested pension liabilities) beliefen sich durchschnittlich auf $34 \%$, die Vermögenswerte der Pensionsfonds auf durchschnittlich $26 \%$ der Nettosubstanzwerte der untersuchten Unternehmen; siehe JOHNSON/HIGGINS, Funding Costs and Liabilities of Large Corporate Pension Plans, 1979, zitiert nach: TEPPER, Taxation and Corporate Pension Policy, The Journal of Finance, 1981, 1. Zur bilanziellen Behandlung der Pensionsaufwendungen in den USA siehe KIESO/WEYGANDT, aaO (Fn. 23), S. 933 ff; in Großbritannien: LEE, aa0 (Fn. 19) S. 88 ff. Zur wirtschaftlichen Bedeutung des Pensionsaufwands und der Pensionsfonds vgl. BULOW, What are Corporate Pension Liabilities? Quarterly Journal of Economics, 1982, 435 ff; FELDSTEIN/SELIGMAN, Pension Funding, Share Prices, and National Savings, The Journal of Finance, 1981, 801 ff; TREYNOR, The Principles of Corporate Pension Finance, The Journal of Finance, 1977, $627 \mathrm{ff}$.

43. Vgl. JUNG, aaO (Fn. 18) S. 182; sowie MÜLLER, ZfbF 1982, 249, 254 f.

44. Vgl. BUSSE VON COLBE, Bilanzen, 4. Aufl., 1981, S. 834; MEYER, Der Nachweis der Ertragslage von Konzernen mit Hilfe konsolidierter Jahresabschlüsse, WPg 1975, 257 ff. Diese Berechnung des „Konzerneigenkapitals" ist eine Erweiterung der Eigenkapitalberechnung für die Einzelgesellschaft nach $\S \S 151,152$ AktG, wie sie in den meisten Standardwerken beschrieben ist: Vgl. COENENBERG, aaO (Fn. 21), S.363; Wirtschaftsprüfer-Handbuch, aaO (Fn. 29), S. 1495; WÖHE, Bilanzierung und Bilanzpolitik, 5. Aufl., 1979.

45. BUSSE VON COLBE, aaO (Fn. 44), S. 835; sowie BUSSE VON COLBE/ORDELHEIDE, aaO (Fn. 31), S. 234 f; vgl. auch KÜTING, Konsolidierungspraxis, 2. Aufl., 1981, S. 209.

46. Die Konsolidierungspraxis einiger Konzerne ist noch nicht eindeutig; vgl. BUSSE VON COLBE/ORDELHEIDE, aaO (Fn. 31), S. 234 Fn. 12.

47. Siehe JUNG, aaO (Fn. 18), S. 284; LEE, aaO (Fn. 19) S. 346 f.

48. BUSSE VON COLBE, aaO (Fn. 44), S. 834; BUSSE VON COLBE/ORDELHEIDE, aaO (Fn. 31), S. 120 f; KÜTING, aaO (Fn. 45), S. 206.

49. BUSSE VON COLBE, aaO (Fn. 44), S. 835.

50. Vgl. COENENBERG, aaO (Fn. 21), S. 363; Wirtschaftsprüfer-Handbuch, aaO (Fn. 29), S. 1495.

51. Vgl. beispielsweise COENENBERG, aaO (Fn. 21), S. 365. Bei den britischen Bilanzen muß zuvor die Saldierung der Current Assets mit den Current Liabilities rückgängig gemacht werden, die zu einer starken Verkürzung der Bilanzsumme führt.

52. Die in den nachfolgenden Tabellen aufgeführten durchschnittlichen Eigenkapitalquoten ergeben sich als die arithmetischen Mittel der (korrigierten) Eigenkapitalquoten der einzelnen Unternehmen des jeweiligen Landes.

53. Vgl. die Ausführungen zu diesen Positionen in den Abschnitten III 1 a), III 2 und III 3.

54. Für Großbritannien errechnet sich die neue Eigenkapitalquote als:

$\mathrm{EQ} 2$ - (EK1 - IAV - NEU - SRE) / (GK1 - IAV - NEU - SRE);

für die USA ergibt sich der erste Wert als

$\mathrm{EQ} 2=(\mathrm{EK} 1-\mathrm{IAV}) /(\mathrm{GK} 1-\mathrm{IAV})$ und der zweite Wert als:

$\mathrm{EQ} 2=(\mathrm{EK} 1-\mathrm{IAV}-0,5 \times \mathrm{FAV}) /(\mathrm{GK} 1-\mathrm{IAV}-0,5 \times \mathrm{FAV})$.

55. Hierunter soll nur der auf Abschreibungsunterschieden basierende Anteil der bilanziell ausgewiesenen "deferred taxes" bei den untersuchten Unternehmen verstanden werden (vgl. Abschnitt III 1 b).

56. Vgl. Abschnitt III 1 b) und SCHMIDLE, aa0 (Fn. 22), S.127 ff.

57. Vgl. Abschnitt III 1 b).

58. Für Großbritannien gilt: EK2 $=$ EK1 - IAV- NEU - SRE; für die USA: EK2 = EKI - IAV $-0,5 \times$ FAV.

59. Für Großbritannien ist GK2 = GK1 - IAV - NEU - SRE; für die USA ist GK2 = GK1 - IAV - 0,5 x FAV.

60. Über die Höhe der ,potentiellen deferred taxes“ ist in den Erläuterungen zu den britischen Bilanzen zu berichten (vgl. Fn. 26 m.w.N.). Allerdings wird der Begriff nicht immer eindeutig verwendet. Hier wird unter "potentiellen deferred taxes“ lediglich der auf Abschreibungsunterschieden beruhende Anteil der „deferred taxes“ verstanden, der die (ebenfalls aus Abschreibungsunterschieden resultierenden) „bilanziell ausgewiesenen deferred taxes“ übersteigt.

61. $\triangle$ SAVP ist der Teil des Abschreibungsunterschiedes zwischen Handels- und Steuerbilanz, für den die „potentiellen deferred taxes“ nicht passiviert werden; vgl. Abschnitt III 1b).

62. $\mathrm{AaO}$ (S. 30); vgl. zu den steuerlichen Abschreibungsmöglichkeiten: BUTTERWORTHS, aaO (Fn. 38), S. 238 ff; FISCHER, DB 1984, 357, 361 ff; FUEST/KROKER, Investitionsbesteuerung im internationalen Vergleich, iw-trends 4 (1983), 28 ff; Institut für Finanzen und Steuern, Steuerrechtliche Behandlung von Investitionen in der Europäischen Gemeinschaft, Grüner Brief Nr. 190, 1980, 56 ff.

63. Vgl. LINDENBERG/ROSS, Tobin's q Ratio and Industrial Organization, Journal of Business, 1981, $1 \mathrm{ff}$.

64. Siehe Statistisches Jahrbuch 1981 für die Bundesrepublik Deutschland S. 705.

65. SCHLESINGER, ZfbF 1984, 6, 9. 
66. Vgl. SCHLESINGER, ZfbF 1984, 6, 9; sowie BUCH, Handelsblatt vom 13./14.1.1984, 13. Demgegenüber schlagen THORMÄHLEN/MICHALK, Wirtschaftsdienst 1983, 87, 88, vor, „Eigenkapital und Pensions-rückstellungen zur Bilanzsumme in Beziehung zu setzen”. Ebenso weist das Rheinisch-Westfälische Institut für Wirtschaftsforschung auf die „,- häufig als graues Eigenkapital apostrophierten - Rückstellungen" hin, aaO (Fn. 2), S. 149.

67. Siehe MALCA, Pension Funds and Other Institutional Investors, Lexington, Massachusetts 1975, S. 10.

68. TEPPER, aaO (Fn.42), S. 8; vgl. auch KOTLIKOFF/SMITH, Pensions in the American Economy, Chicago 1983, S. 315, nach denen die Aktien im Jahre 1980 sogar 58,7 \% des zu Marktwerten bewerteten Portfolios der "private pension funds" ausmachten.

69. Vgl. KIESO/WEYGANDT, aaO (Fn. 23), S. 935 Fn. 5. In Großbritannien hielten die Pensionsfonds 1973 ca. $13 \%$ der Aktien, vgl. The Economist Intelligente Unit Special Report No. 43, Pension Funds in the UK, London 1977, S.101 f.

70. Vgl. FLASSBECK/KOLL, Wirtschaftswoche Nr. 21, 1983, 72, 76.

71. SCHOENFELD, Grundzüge der Rechnungslegung in den USA, ZfB 1981, 290, 303.

72. Zur Bildung von Rückstellungen in Deutschland vgl. HEINEN, Handelsbilanzen, 10. Aufl., 1982, S. 295 f; in den USA: JUNG, aaO (Fn. 18), S. $164 \mathrm{ff}$.

73. Vgl. SCHOENFELD, ZfB 1981, 290, 303; siehe auch MÜLLER, ZfbF 1982, 249, $254 \mathrm{f}$.

74. Als Indiz für diese Angleichung kann auch der Abschluß der BASF AG gelten. Die Umrechnung des deutschen Gruppenabschlusses für das Jahr 1980 auf der Grundlage der US-amerikanischen Bilanzierungsgrundsätze führte zu einer Erhöhung des ausgewiesenen Eigenkapitals um $24 \%$. Der Eigenkapitalanteil am Gesamtkapital stieg von rund $42 \%$ auf nahezu $50 \%$ an und erreichte damit den für die USA üblichen Eigenkapitalanteil. Vgl. Geschäftsbericht der BASF AG 1980, S. 47.

75. GRUHLER, Die AG 1984, 100. 